\title{
Physics from the S-matrix: Scattering Amplitudes without Lagrangians
}

\author{
Eduardo Conde* \\ Physique Théorique et Mathématique, Université Libre de Bruxelles and International Solvay \\ Institutes \\ E-mail: econdepe@ulb.ac.be
}

The aim of these lectures is to provide a rigorous background for someone interested in the socalled on-shell methods for the computation of scattering amplitudes. With the hindsight gained by the exciting recent advances in the field, we revisit the essential ideas of the "S-matrix program", putting the emphasis on their proof (and what are the assumptions needed at each step) rather than on their utilization. Although part of the discussion is valid at any order in perturbation theory, we focus mostly on the tree level, and briefly introduce the BCFW recursion relations as an example of on-shell methods.

Ninth Modave Summer School in Mathematical Physics,

1-7 September, 2013

Modave, Belgium

\footnotetext{
${ }^{*}$ Speaker.
} 


\section{Contents}

1. Formalities. The amplitude as a formal object 4

1.1 Definitions and notation 4

1.2 Brief review of irreducible representations of the Poincaré group 5

$\begin{array}{lll}1.3 & \text { More about one-particle states } & 6\end{array}$

$\begin{array}{lll}1.4 & \text { The S-matrix } & 7\end{array}$

1.5 Connectedness structure and the cluster decomposition principle 9

$\begin{array}{lll}1.6 & \text { Unitarity and factorization } & 11\end{array}$

$\begin{array}{lll}1.7 \text { Crossing symmetry } & 16\end{array}$

$\begin{array}{llr}1.8 \text { Soft limits } & 18\end{array}$

$\begin{array}{lll}1.9 & \text { Reconstructing the amplitude at tree level } & 20\end{array}$

2. Building tree-level amplitudes without Lagrangians 20

$\begin{array}{lll}2.1 & \text { BCFW and generalizations } & 23\end{array}$

2.2 Massless kinematics in four dimensions $\quad 28$

2.3 Three-particle amplitudes $\quad 32$

2.4 Three neat examples on the use of BCFW 35

2.4.1 Self-interacting particle of spin $s \quad 35$

$\begin{array}{lll}\text { 2.4.2 Parke-Taylor formula } & 38\end{array}$

2.4.3 The four-particle test 42

3. To loops... and beyond! 43

\section{Foreword}

Although no unpublished results are included in these notes, they should not be regarded as a comprehensive review. Consequently, many interesting and important references have been left out, and only the publications presumably relevant for the material presented are cited along the way. To the authors of the former, I apologize for not having space to include their work. Since we are revisiting the S-matrix program, an obvious heavy influence to these notes is [1]. The reader will also find that Weinberg's Quantum Field Theory (QFT) book [2] also played a big role in preparing these lectures. For the reader interested in a real review covering similar material, several appropriate and interesting references have come out lately: for instance the exhaustive [3] or the less conventional [4]. 


\section{Motivation}

My motivation to give this course was to critically analyze the assumptions that underly (some of) the so-called on-shell methods. Since we grow accustomed to think of QFT in Lagrangian terms, it is easy to take for granted certain notions which are automatic within the standard QFT apparatus, but may be not in a different formalism. And it is hard sometimes to decouple these notions from their Lagrangian origin. If we really want to advocate a new (old, really) point of view, we should be able to embrace it in a self-consistent manner. Also, I have found that typically not so much attention is paid to these matters in the literature. Maybe with the good reason that anyway we know many properties are correct from our experience with standard QFT, and it is more interesting to look for unknown phenomena rather than soundly establishing the known ones. However, if one is to go beyond and generalize, in some sense, standard QFT, it is important to have good control on the foundations. Roughly, the endpoint of these notes coincides with the starting point of any of the two reviews cited above. So the target reader is a person interested in that "below-zero" knowledge.

Otherwise, the notes can be taken as a (probably too formal) short introduction to some of the on-shell methods for scattering amplitudes. The basic motto of these methods is "to compute amplitudes without Lagrangians".

Why focus on scattering amplitudes? Well, the main experimental access we have to high energy physics is through the experiments done at the big colliders. What one does is to accelerate two beams of particles until they have a huge kinetic energy, and then let them collide. Next we observe the leftovers of this collision, and infer the physics going on. This might seem like an intricate way of doing experiments, but the nature of Quantum Mechanics, where measurements cannot be made without affecting the result, does not leave room for many other options.

While very useful, and the only way we understand QFT so far, a Lagrangian contains a lot of off-shell structure, that is useless when computing scattering amplitudes. If we think about the path integral formulation, this off-shell structure encodes all the information needed to compute the action of a particle traveling any path away from the one dictated by the equations of motion. Then one averages over all these paths to get the final answer. This is an intuitive picture, but a very impractical one, since at the end we only care about on-shell (observable) quantities. Let us illustrate this with an example: the Lagrangian for the Yang-Mills (YM) theory has two interaction vertices, a three-point vertex, and a four-point one. It turns out that the three-point one contains enough information to compute any $n$-point amplitude. The four-point vertex contains no physical information, its only mission being to guarantee gauge invariance off-shell. The situation is even more spectacular for Gravity (GR), which is a fine QFT at tree level, because the Lagrangian contains an infinite number of interaction vertices, but only the three-point one is relevant for the computation of scattering amplitudes!

So how do we intend to compute amplitudes if we do not have a Lagrangian? The plan is to build the S-matrix just from a series of postulates. The idea is not new, and it went under the name of S-matrix program in the 60's. What will be new are the tools we have nowadays to address this line of thinking. Our axioms will be:

1. Poincaré invariance. We try to describe scattering in flat Minkowski space-time. Actually we just need the space to be asymptotically flat. 
2. Existence of asymptotic one-particle states. They describe the particles we scatter. They are in correspondence with the irreducible representations of the Poincaré group.

3. Analyticity. We require that the S-matrix is analytic in the external momenta, as we continue them to complex values.

4. Cluster decomposition principle. It gives a weak notion of locality, by which we understand that all singularities of the S-matrix come from propagators.

With these axioms in mind, we will build the S-matrix, and discuss its formal properties plus part of its singularity structure in Section 1 . Then we move in Section 2 to the question of how to reconstruct the tree-level part of the S-matrix from the axioms above. Let us get started!

\section{Formalities. The amplitude as a formal object}

Although we will not define the scattering amplitude until Section 1.5, let us make already a couple of remarks about it. As any other QFT observable, we will think of it perturbatively. If the theory has a dimensionless coupling constant $g$, then the scattering amplitude for $n$ external legs $M_{n}$ admits a perturbative expansion

$$
M_{n}(g)=g^{n_{\text {tree }}} M_{n}^{\text {tree }}+g^{n_{1-\text { loop }}} M_{n}^{1-\text { loop }}+g^{n_{2-\text { loop }}} M_{n}^{2-\text { loop }}+\cdots .
$$

Presumably, this series is asymptotic and $M_{n}$ is only well defined when we include the nonperturbative (instantonic, i.e. proportional to $e^{-\# / g^{2}}$ ) corrections, but we will not care about any of these in these lectures. The exponents $n_{\text {tree }}, n_{1-\text { loop }}$, etc depend on the theory. For theories with a three-particle vertex, $n_{\mathrm{L}-\text { loop }}=g^{n-2+2 L}$ (the tree level is understood as $L=0$ loops). So, for a fixed number of external legs $n$, notice that the loop order is just determined by the power of the coupling constant that accompanies the amplitude. Of course, every loop order will be characterized by a different singularity structure, but there is no need to think of the loop as a real loop in a Feynman diagram.

Notice also that the power of $g$ that comes with the $L$-loop $n$-point amplitude grows either when we have more loops or either when we increase the number of external legs. In a certain sense, going up in the number of external particles is the same as going up in the number of loops.

\subsection{Definitions and notation}

We start working in $D+1$ dimensions (later in Section 2 we will restrict to $D=3$ ) and we assume an (asymptotic ${ }^{1}$ ) space-time with isometries given by the Poincaré group $\mathbb{R}^{1, D} \ltimes \mathrm{SO}(1, D)$. We use a $(-,+, \ldots,+)$ signature and units where $c=1, \hbar=1$, so that $p^{2}=-m^{2}$ on the mass-shell. The generators of the Poincaré algebra are denoted by $P^{\mu}$ (four translations) and $J^{\mu v}$ (Lorentz transformations: three boosts and three spatial rotations). Additionally, we denote the complex conjugate of $z$ by $z^{*}$.

We want to have quantum-mechanical particles, so we also assume that asymptotic oneparticle states exist, and that they form the basis of our Hilbert space. We denote them by $|p, a\rangle$ out,

\footnotetext{
${ }^{1}$ Only the asymptotic properties of the space-time are needed for the discussion, since the way to conceive a scattering process is by preparing an initial state at past infinity, and observing it at future infinity.
} 
where $p$ is a $(D+1)$-momentum, and $a$ stands for a set of quantum labels, as for instance the charge, spin/helicity, etc. Because of the causal structure of the asymptotic space-time, we have to distinguish between in and out states. When this distinction is not important, we will drop the label. The defining property that these one-particle states verify is

$$
P^{\mu}|p, a\rangle=p^{\mu}|p, a\rangle
$$

Under a Poincaré transformation they must transform as

$$
U(\Lambda)|p, a\rangle=\sum_{a^{\prime}} D(\Lambda, k)_{a a^{\prime}}\left|\Lambda p, a^{\prime}\right\rangle, \quad \Lambda \in \mathbb{R}^{1, D} \ltimes \mathrm{SO}(1, D),
$$

where the $D(\Lambda, k)_{a a^{\prime}}$ furnish a representation of the Poincaré group . When this representation is irreducible, we talk about an elementary particle. Therefore, elementary particles are in correspondence with irreducible representations of $\mathbb{R}^{1, D} \ltimes \mathrm{SO}(1, D)$. For $D=3$ these were first studied in the classical paper by Wigner [5]. His method can be straightforwardly extended to generic $D>2$. Of course the Wigner classification can be found in [2], and one can also have a look at [6] for a nice recent review. We quickly go over it here for completeness.

\subsection{Brief review of irreducible representations of the Poincaré group}

We recall how one determines the possible matrices $D(\Lambda, k)_{a a^{\prime}}\left|\Lambda p, a^{\prime}\right\rangle$ that we can use in equation (1.3). The translations constitute an Abelian subgroup of the full Poincare group, and their action is determined by (1.2). They act on the one-particle states by just adding a phase:

$$
\Lambda: x^{\mu} \rightarrow x^{\mu}+z^{\mu} \Longrightarrow U(\Lambda)=e^{-i z \mu P^{\mu}}, \quad U(\Lambda)|p, a\rangle=e^{-i z \mu p^{\mu}}|p, a\rangle .
$$

Regarding the Lorentz transformations ${ }^{2}$, some of them can be chosen to act trivially. For each class of momentum $p$, we choose a reference one $p_{\text {ref }}$ (for example the standard choices for massive and light-like particles are $p_{\text {ref }}=(m, 0,0,0)$ and $p_{\text {ref }}=(E, E, 0,0)$ respectively $)$ and a reference Lorentz transformation such that $p=L(p) p_{\text {ref. }}$ Then we define:

$$
|L(p) p, a\rangle=U(L p)|p, a\rangle .
$$

We would be done if it were not for the fact that there are Lorentz transformations that do not alter the $(D+1)$-momentum $\Lambda p=p$. These transformations might nonetheless alter the physical state:

$$
U(\Lambda)|p, a\rangle=\sum_{a^{\prime}} D(\Lambda, p)_{a a^{\prime}}\left|p, a^{\prime}\right\rangle
$$

They conform the so-called Little Group $L G_{p}$ (the fancy name for the stabilizer of the Lorentz group). Although it depends on the momentum $p$, we have that for $p \neq 0$ :

$$
L G_{p} \cong\left\{\begin{array}{ll}
\operatorname{ISO}(D-1) & \text { for massless particles } \\
\mathrm{SO}(D) & \text { for massive particles }
\end{array} .\right.
$$

\footnotetext{
${ }^{2}$ We actually restrict to the proper orthochronous component of the Lorentz group. The study of the other components would entail the study of the $\mathrm{P}$ and $\mathrm{T}$ operations, which will not be relevant for our purposes.
} 
Therefore, the irreducible representations of the Lorentz group are in correspondence with the irreducible representations of the Little Group. In the massive case, the representations of the orthogonal groups are well-known, and $a$ is a Young tableau that we will call a "spin" index, by analogy with the case $D=3$. For the massless case, $\operatorname{ISO}(D-1)$ is the group of isometries of $\mathbb{R}^{D-1}$, and it is a non-compact group: $\operatorname{ISO}(D-1)=\mathbb{R} \ltimes \operatorname{SO}(D-2)$. Typically one is only concerned with the $\mathrm{SO}(D-2)$ part. In that case, the label $a$ is discrete and it is another (smaller) Young tableau that we will call a "helicity" index. Otherwise it can be continuous, and gives rise to the so-called continuous-spin representations. In four dimensions, these representations are called continuousspin particles (CSP).

Little group in four dimensions The Lorentz group in four dimensions has six generators $J^{\mu \nu}$. The Little Group $L G_{p}$ has dimension three and it is generated by the independent components of the Pauli-Lubanski vector

$$
W^{\mu}=\frac{1}{2} \varepsilon^{\mu \nu \rho \sigma} p_{v} J_{\rho \sigma}
$$

which are three given that $p \cdot W=0$ by construction. From the Poincare algebra it follows that

$$
\left[W^{\mu}, W^{v}\right]=-i \varepsilon^{\mu v \rho \sigma} W_{\rho} P_{\sigma}
$$

For massive particles one can choose $p=(m, 0,0,0) \Longrightarrow W=m\left(0, J^{23}, J^{31}, J^{12}\right)$ and the Little Group is obviously $\mathrm{SO}(3)$. For massless particles we can choose $p=(E, E, 0,0)$ and $W^{\mu}=$ $-p^{\mu} R+\varepsilon_{1}^{\mu} T_{1}+\varepsilon_{2}^{\mu} T_{2}\left(\varepsilon_{1,2} \cdot p=0\right)$, where the commutation relations (1.9) imply that $T_{1}$ and $T_{2}$ are translations in a two-dimensional Euclidean plane, and $R$ is the rotation in this plane. So the Little Group in this case is $\operatorname{ISO}(2)$.

If we forget about the non-compact part (i.e. we assume it acts trivially), we see that in $3+1$ dimensions the Little Group for massless particles is Abelian: $\mathrm{SO}(2)=\mathrm{U}(1)$, so the irreducible representations are one-dimensional and characterized by an semi-integer $h$, called helicity. Writing a Lorentz transformation as $\Lambda=L(p) e^{i\left(\beta_{1}(\Lambda, p) T_{1}+\beta_{2}(\Lambda, p) T_{2}+\theta(\Lambda, p) R\right)}$, it is clear that it will act on a one-particle state by adding a phase:

$$
U(\Lambda)|p, h\rangle=e^{i h \theta(\Lambda, p)}|p, h\rangle \quad \Leftrightarrow \quad D(\Lambda, p)_{a a^{\prime}}=e^{i h \theta(\Lambda, p)} \delta_{a a^{\prime}}
$$

The number $h$ is called helicity and it is an integer for bosons and a semi-integer for fermions (in this case one should consider representations of $\operatorname{Spin}(2)$ than of $\mathrm{SO}(2)$ ). Notice that a particle is associated with a certain helicity $h$. Different helicities give different particles. When we say that a massless particle in four dimensions has two possible helicities, it is because CPT invariance relates the $+h$ particle with the $-h$ one.

\subsection{More about one-particle states}

We choose to normalize the one-particle states as

$$
\left\langle p^{\prime}, a^{\prime} \mid p, a\right\rangle=(2 \pi)^{D} 2 p^{0} \delta^{(D)}\left(\vec{p}-\vec{p}^{\prime}\right) \delta_{a a^{\prime}} .
$$

This normalization, typically called relativistic normalization, may seem a bit cumbersome; indeed it is not the one Weinberg uses in [2], but it is more friendly to covariance: if $k=\Lambda p$ and $k^{\prime}=\Lambda p^{\prime}$ 
then $2 k^{0} \delta^{(D)}\left(\vec{k}-\vec{k}^{\prime}\right)=2 p^{0} \delta^{(D)}\left(\vec{p}-\vec{p}^{\prime}\right)$ (this can be easily proved from the invariant Lorentz measure $\left.\int \mathrm{d}^{D+1} p \delta\left(p^{2}+m^{2}\right) \boldsymbol{\theta}\left(p^{0}\right)=\int \frac{\mathrm{d}^{D} \vec{p}}{2 p^{0}}\right)$.

Certainly in a free theory the one-particle states would be built by acting with creation operators $\mathfrak{a}^{\dagger}(p, a)$ on the vacuum $|0\rangle:|p, a\rangle_{\text {free }}=\mathfrak{a}^{\dagger}(p, a)|0\rangle$. This is no longer the case in an interacting theory, and one could wonder how the states in the latter are related to free states. This question is not very relevant for our purposes since we assume that the one-particle states exist with the properties we have mentioned. However, for the interested reader, it is addressed in chapter 3.1 of [2]. There, the idea that "asymptotically, interacting states are free states" is made formal.

A basis for the full Hilbert space of physical states (or Fock space) is given by combining single-particle states $|p, a\rangle$ into multi-particle ones, that we will generically denote with greek letters $|\alpha\rangle$ :

$$
|\alpha\rangle=\left|p_{(1)}, a_{(1)}\right\rangle \otimes \cdots \otimes\left|p_{\left(n_{\alpha}\right)}, a_{\left(n_{\alpha}\right)}\right\rangle=\left|\left\{p_{(i)}, a_{(i)}\right\}\right\rangle .
$$

If we permute the one-particle states that form $|\alpha\rangle$ with a permutation $\sigma \in S_{n_{\alpha}}$, then $|\alpha\rangle \rightarrow$ $(-1)^{S_{\sigma}}|\alpha\rangle$, where $S_{\sigma}$ is equal to 0 (1) if an even (odd) number of fermions have been interchanged by it. Sometimes it will be convenient talk about the momentum of a multi-particle state, which we will indicate as

$$
p_{(\alpha)}=\sum_{i=1}^{n_{\alpha}} p_{(i)} .
$$

The normalization of these multi-particle states follows from (1.11):

$$
\left\langle\alpha^{\prime} \mid \alpha\right\rangle=\delta_{n_{\alpha} n_{\alpha^{\prime}}}(2 \pi)^{2 D n_{\alpha}} \sum_{\sigma}(-1)^{S_{\sigma}} \prod_{i=1}^{n_{\alpha}} 2 p_{(i)}^{0} \delta^{(D)}\left(\vec{p}_{(i)}-{\overrightarrow{p^{\prime}}}_{(\sigma(i))}\right) \delta_{a_{(i)} a_{(\sigma(i))}^{\prime}} \equiv \delta\left(\alpha-\alpha^{\prime}\right) .
$$

It is also obvious from (1.3) how these states should transform under a Poincaré transformation, characterized by a translation by $z$ and a Lorentz rotation $\Lambda$ :

$$
U(\Lambda, z)\left|\left\{p_{(i)}, a_{(i)}\right\}\right\rangle=e^{-i z \mu p_{(\alpha)}^{\mu}} \sum_{a_{(1)}^{\prime}, \cdots, a_{\left(n_{\alpha}\right)}^{\prime}} D\left(\Lambda, p_{(1)}\right)_{a_{(1)} a_{(1)}^{\prime}} \cdots D\left(\Lambda, p_{\left(n_{\alpha}\right)}\right)_{a_{\left(n_{\alpha}\right)} a_{\left(n_{\alpha}\right)}^{\prime}}\left|\left\{\Lambda p_{(i)}, a_{(i)}^{\prime}\right\}\right\rangle .
$$

Before moving on to the definition of the S-matrix, it is important to write the unity of the Hilbert space in the basis of multi-particle states. It is given by

$$
\mathbb{1}=|0\rangle\left\langle 0\left|+\sum_{n_{\alpha}=1}^{\infty} \frac{1}{(2 \pi)^{D n_{\alpha}}} \int \prod_{i=1}^{n_{\alpha}} \frac{\mathrm{d}^{D} \vec{p}_{(i)}}{2 p_{(i)}^{0}} \sum_{a_{(i)}}\right|\left\{p_{(i)}, a_{(i)}\right\}\right\rangle\left\langle\left\{p_{(i)}, a_{(i)}\right\}\left|\equiv \int_{\alpha} \mathrm{d} \alpha\right| \alpha\right\rangle\langle\alpha| .
$$

\subsection{The S-matrix}

We actually have two basis to describe our Hilbert space of physical states: via the in states $|\alpha\rangle^{\text {in }}$ or via the out states $|\alpha\rangle^{\text {out }}$. The matrix which takes us from the latter basis to the former is called the S-matrix:

$$
S_{\beta \alpha}={ }^{\text {out }}\langle\beta \mid \alpha\rangle^{\text {in }}, \quad \text { or } \quad \mathrm{S}=\int_{\alpha} \mathrm{d} \alpha|\alpha\rangle^{\text {in }}{ }_{\text {out }}\langle\alpha| .
$$

In other words, the S-matrix tells us how to expand an in state in terms of out states. The inverse operation is done with the Hermitian conjugate $S^{\dagger}$ :

$$
S_{\beta \alpha}^{\dagger}={ }^{\text {in }}\langle\beta \mid \alpha\rangle^{\text {out }}=\left(S_{\alpha \beta}\right)^{*}, \quad \text { or } \quad \mathrm{S}^{\dagger}=\int_{\alpha} \mathrm{d} \alpha|\alpha\rangle^{\text {out }}{ }_{\text {in }}\langle\alpha| .
$$


Since the two basis are orthonormal (as can be seen from (1.14), which apply to both in and out states), the S-matrix is unitary by construction:

$$
S S^{\dagger}=\int_{\alpha} \mathrm{d} \alpha \int_{\beta} \mathrm{d} \beta|\alpha\rangle^{\text {in }}{ }_{\text {out }}\langle\alpha \mid \beta\rangle_{\text {out }}{ }^{\text {in }}\left\langle\beta\left|=\int_{\alpha} \mathrm{d} \alpha\right| \alpha\right\rangle^{\text {in }}{ }_{\text {in }}\langle\alpha|=\mathbb{1}=S^{\dagger} S .
$$

Of course, rather than thinking of the S-matrix as a change of basis, we are interested in thinking of its matrix elements as transition amplitudes from certain in states to evolve into certain out states. Let us say that $|\alpha\rangle=\left|\left\{p_{(i)}, a_{(i)}\right\}\right\rangle$ with $i$ ranging from 1 to $n_{\alpha}$, and $|\beta\rangle=\left|\left\{p_{\left(i^{\prime}\right)}^{\prime}, a_{\left(i^{\prime}\right)}^{\prime}\right\}\right\rangle$ with $i^{\prime}$ from 1 to $n_{\beta}$. We will use the following graphical notation for $S_{\beta \alpha}$ and $S_{\beta \alpha}^{\dagger}$ :

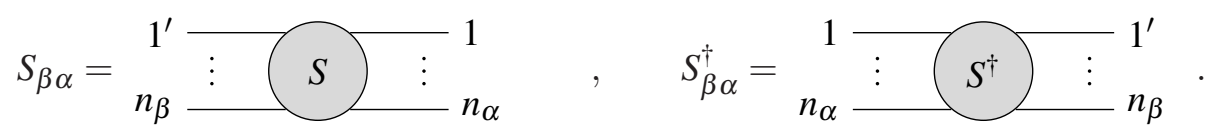

Notice that, contrary to (maybe) the usual intuition, the state at past infinity is represented on the right, while the one at future infinity is represented on the left.

If we were given a Hamiltonian $H=H_{0}+V$, one could write a formal expression for the operator $S$ in (1.17): $S=U(-\infty, \infty)$, where $U\left(\tau, \tau_{0}\right)=e^{i H_{0} \tau} e^{-i H\left(\tau-\tau_{0}\right)} e^{-i H_{0} \tau_{0}}$. Using time-dependent perturbation theory, this formula is the starting point for the derivation of the Feynman diagrammatic expansion of the S-matrix. But we are interested in following a different road, that of determining $S$ from its analytic properties and symmetries, without the need of a Lagrangian.

Symmetries constrain the S-matrix: Any unitary transformation $U$ that acts on the same way in the in and out states imposes constraints on the S-matrix:

$$
{ }^{\text {out }}\langle\beta \mid \alpha\rangle^{\text {in }}={ }^{\text {out }}\langle U \beta \mid U \alpha\rangle^{\text {in }} \text {. }
$$

Such a transformation $U$ is always associated to a conserved symmetry.

Take for instance the Poincaré symmetry,

- if we look at the translations: $U=e^{-i z_{\mu} P^{\mu}}$. Then ${ }^{\text {out }}\langle\beta \mid \alpha\rangle^{\text {in }}={ }^{\text {out }}\left\langle e^{-i z_{\mu} p_{(\beta)}^{\mu}} \beta \mid e^{-i z_{\mu} p_{(\alpha)}^{\mu}} \alpha\right\rangle^{\text {in }}=$

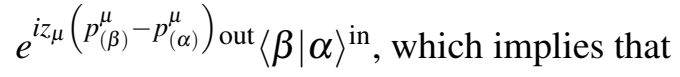

$$
{ }^{\text {out }}\langle\beta \mid \alpha\rangle^{\text {in }} \propto \delta^{(D+1)}\left(p_{(\beta)}-p_{(\alpha)}\right) .
$$

So we deduce from here that the S-matrix must contain a momentum-conserving deltafunction.

- if we focus instead on the Lorentz transformations, from (1.15) we get the condition

$$
\left(\sum_{a_{(j)}^{\prime}} \prod_{j=1}^{n_{\beta}} D\left(\Lambda, p_{(j)}\right)_{a_{(j)} a_{(j)}^{\prime}}\right)^{*}\left(\sum_{a_{(i)}^{\prime}} \prod_{i=1}^{n_{\alpha}} D\left(\Lambda, p_{(i)}\right)_{a_{(i)} a_{(i)}^{\prime}}\right){ }^{\text {out }}\left\langle\beta^{\prime} \mid \alpha^{\prime}\right\rangle^{\text {in }}={ }^{\text {out }}\langle\beta \mid \alpha\rangle^{\text {in }},
$$

where $\left|\beta^{\prime}\right\rangle=\left|\left\{\Lambda p_{(j)}, a_{(j)}\right\}\right\rangle,\left|\alpha^{\prime}\right\rangle=\left|\left\{\Lambda p_{(i)}, a_{(i)}\right\}\right\rangle$.

Any $\mathrm{U}(1)$ symmetry, $U=e^{-i \theta Q}$ would work pretty much like the translations. If we assume that every state $\left|p_{(i)}, a_{(i)}\right\rangle$ has charge $q_{(i)}$ under the transformation, it is immediate to deduce the condition for charge conservation

$$
{ }^{\text {out }}\langle\beta \mid \alpha\rangle^{\text {in }} \propto \delta\left(\sum_{j=1}^{n_{\beta}} q_{(j)}-\sum_{i=1}^{n_{\alpha}} q_{(i)}\right) .
$$




\subsection{Connectedness structure and the cluster decomposition principle}

When the interaction is trivial, $S_{\beta \alpha}=\delta(\alpha-\beta)$. When it is not, $S_{\beta \alpha}$ still contains a $\delta(\alpha-$ $\beta$ ) factor because there is a non-zero probability that the particles will not interact. We are not interested in this trivial part, so we would like to "extirpate" it from the S-matrix. To that end, we define the connected part of the S-matrix, to be denoted by $S^{C}$. We do it recursively:

$$
S_{\beta \alpha}=S_{\beta \alpha}^{C}+\sum_{\text {partitions } \mathscr{P}}(-1)^{S_{\mathscr{P}}} S_{\beta_{1} \alpha_{1}}^{C} \cdots S_{\beta_{r} \alpha_{r}}^{C},
$$

where the $\mathscr{P}$ stand for the partitions of the sets of initial and final particles, and $S_{\mathscr{P}}=1$ if the rearranging $\alpha \rightarrow \alpha_{1} \ldots \alpha_{r}, \beta \rightarrow \beta_{1} \ldots \beta_{r}$ involves rearranging an odd number of fermions (and it is zero otherwise). The beginning of the recursion is given by the obvious fact that

$$
S_{\beta \alpha}^{C}=\delta(\beta-\alpha) \quad \text { when } \beta \text { and } \alpha \text { are 1-particle states. }
$$

The connectedness is best appreciated graphically with some examples. For example for a two-two scattering:

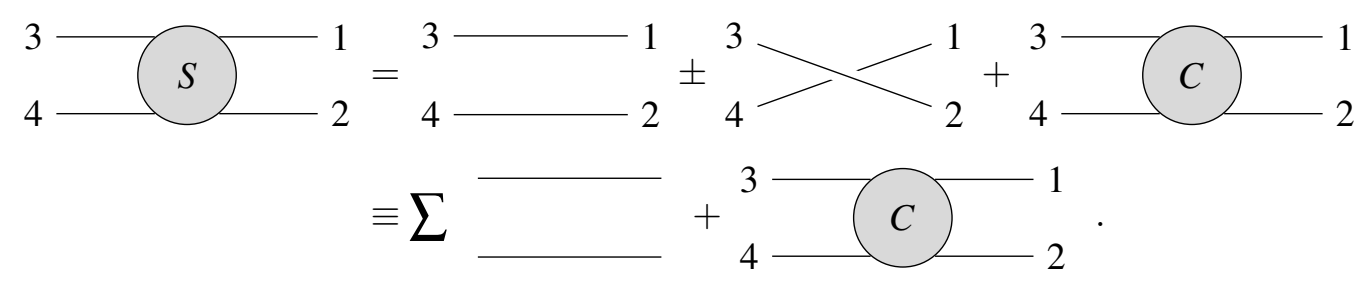

The plus/minus sign on the first line accounts for the possibility that we are interchanging some fermions (for instance we should use the minus sign if particles 3 and 4 are fermions). To shorthand the notation, on the second line we must understand that under the sum sign we group the two first terms on the first line.

So far we are doing nothing else than rewriting $S_{\beta \alpha}$, and parameterizing it in a convenient way. If we want to introduce some physical input, we can do it via the cluster decomposition principle . In this language it is stated as:

$$
S_{\beta \alpha}^{C}=0 \quad \text { when at least one of the particles is far from the others. }
$$

This statement is formulated in position space. If we Fourier-transform,

$$
S_{x, \beta \alpha}^{C}=\frac{1}{(2 \pi)^{D\left(n_{\alpha}+n_{\beta}\right)}} \int \prod_{i=1, j=1}^{n_{\alpha}, n_{\beta}} \mathrm{d}^{D} \vec{p}_{(i)} \mathrm{d}^{D} \vec{p}_{(j)} e^{-i \sum_{i}^{n \alpha} \vec{p}_{(i)} \cdot \vec{x}_{(i)}+i \sum_{j}^{n_{\beta}} \vec{p}_{(j)} \cdot \vec{x}_{(j)}} S_{\beta \alpha}^{C},
$$

we can see that the cluster decomposition principle imposes conditions on how smooth $S_{\beta \alpha}^{C}$ should be. Loosely speaking, the cluster decomposition principle implies that $S_{\beta \alpha}^{C}$ should not contain $\delta$-functions other than $\delta^{(D+1)}\left(p_{(\beta)}-p_{(\alpha)}\right)$.

We now define the scattering amplitude, $M_{\beta \alpha}$ for the process $\alpha \rightarrow \beta$, by

$$
S_{\beta \alpha}^{C} \equiv i(2 \pi)^{D+1} \delta^{(D+1)}\left(p_{(\beta)}-p_{(\alpha)}\right) M_{\beta \alpha} \text {. }
$$


From the normalization condition (1.11), we can read the mass dimension $[|p, a\rangle]=\frac{1-D}{2}$. Then from the definition of the S-matrix (1.17) we obviously have that $\left[S_{\beta \alpha}\right]=\frac{n_{\alpha}+n_{\beta}}{2}(1-D)$, if $|\alpha\rangle$ $(|\beta\rangle)$ contains $n_{\alpha}\left(n_{\beta}\right)$ particles. Therefore the mass dimension of the scattering amplitude in $D+1$ dimensions is

$$
\left[M_{\beta \alpha}\right]=D+1+\frac{n_{\alpha}+n_{\beta}}{2}(1-D) .
$$

The scattering amplitude $M$ inherits the Lorentz transformation properties of the S-matrix (1.23) :

$$
\left.M_{\beta \alpha}=\left(\sum_{a_{(j)}^{\prime}} \prod_{j=1}^{n_{\beta}} D\left(\Lambda, p_{(j)}\right)_{a_{(j)}} a_{(j)}^{\prime}\right)\right)^{*}\left(\sum_{a_{(i)}^{\prime}} \prod_{i=1}^{n_{\alpha}} D\left(\Lambda, p_{(i)}\right)_{a_{(i)} a_{(i)}^{\prime}}\right) M_{\beta^{\prime} \alpha^{\prime}} .
$$

We should also remark that if we interchange two particles in $\alpha$ or $\beta$, the amplitude flips the sign if $|\alpha\rangle$ of $|\beta\rangle$ do, which happens when those two particles are fermions. Otherwise it remains invariant. Notice that a scattering amplitude must always contain an even number of fermions.

Uses of amplitudes: Although the purpose of the lectures is to convince the reader of the utility of scattering amplitudes as a theoretical probe of QFTs, their traditional (and still eminent nowadays) use is for experimental purposes. They give us the paramount cross sections (or decay rates in case the initial state is made of only one particle). Essentially, "the cross section is the square of the scattering amplitude". Without looking for rigor, the exact factors of proportionality can be quickly derived by assuming Minkowski space is a $D$-dimensional box of volume $V$, times a time slice of length $T$ :

We normalize the states as

$$
|\alpha\rangle \rightarrow|\alpha\rangle_{\mathrm{box}}=\frac{1}{\sqrt{2 p^{0} V}}|\alpha\rangle .
$$

The orthonormality condition (1.11) becomes

$$
{ }_{\text {box }}\left\langle p^{\prime}, a^{\prime} \mid p, a\right\rangle_{\text {box }}=\frac{1}{2 V \sqrt{p^{0} p^{\prime 0}}}\left\langle p^{\prime}, a^{\prime} \mid p, a\right\rangle=\frac{(2 \pi)^{D}}{V} \delta^{(D)}\left(\vec{p}-\vec{p}^{\prime}\right) \delta_{a a^{\prime}}=\delta_{\vec{p} \vec{p}^{\prime}} \delta_{a a^{\prime}},
$$

where in the last line we have used that in a finite volume

$$
\delta^{(D)}\left(\vec{p}-\vec{p}^{\prime}\right)=\frac{1}{(2 \pi)^{D}} \int \mathrm{d}^{D} \vec{x} e^{i\left(\vec{p}-\vec{p}^{\prime}\right) \cdot \vec{x}}=\frac{V}{(2 \pi)^{D}} \delta_{\vec{p} \vec{p}^{\prime}}
$$

Now, the probability that a state $\alpha$ evolves into a state $\beta$ in this boxed Minkowski space is simply

$$
P(\alpha \rightarrow \beta)=\left|\underset{\text { box }}{\text { out }}\langle\beta \mid \alpha\rangle_{\text {box }}^{\text {in }}\right|^{2}=\frac{1}{\prod_{\beta, \alpha} 2 p_{(k)}^{0} V}\left|S_{\beta \alpha}\right|^{2} .
$$

Assuming that no final and initial momenta are equal (so that $S_{\beta \alpha}=S_{\beta \alpha}^{C}$ ), we can use the definition (1.29) to express

$$
\left|S_{\beta \alpha}\right|^{2}=(2 \pi)^{2(D+1)}\left(\delta^{(D+1)}\left(p_{(\beta)}-p_{(\alpha)}\right)\right)^{2}\left|M_{\beta \alpha}\right|^{2}=(2 \pi)^{(D+1)} \delta^{(D+1)}\left(p_{(\beta)}-p_{(\alpha)}\right) V T\left|M_{\beta \alpha}\right|^{2} .
$$

So it is the modulus squared of $M_{\beta \alpha}$ that gives the probability for the scattering. In order to extract a cross section from here, we have to think how one does measures in the lab. Of course a single 
state is "infinitely thin" in the spectrum of possible measures. Instead of detecting a single final state, we rather sweep a small region of momenta $\prod_{\beta} \mathrm{d}^{D} \vec{p}_{(j)}$. Being careless about the possibility of quantum numbers, we call this infinitesimal region $\mathrm{d} \beta$, with some factors that will be clear from equation (1.37). Since a quantum particle in a box has its momentum quantized with a density $\frac{(2 \pi)^{D}}{V}$, the number of states $\mathrm{d} N_{\beta}$ in $\mathrm{d} \beta$ is

$$
\mathrm{d} N_{\beta}=\left(\frac{V}{(2 \pi)^{D}}\right)^{N_{\beta}} \prod_{\beta} \mathrm{d}^{D} \vec{p}_{(j)}=\left(\prod_{\beta} 2 p_{(j)}^{0} V\right) \mathrm{d} \beta,
$$

and we measure the infinitesimal probability

$$
\mathrm{d} P(\alpha \rightarrow \beta)=P(\alpha \rightarrow \beta) \mathrm{d} N_{\beta}
$$

Putting together the last four equations, we get

$$
\mathrm{d} P(\alpha \rightarrow \beta)=\frac{(2 \pi)^{D+1}}{\prod_{\alpha} 2 p_{(i)}^{0} V} V T \delta^{(D+1)}\left(p_{(\beta)}-p_{(\alpha)}\right)\left|M_{\beta \alpha}\right|^{2} \mathrm{~d} \beta .
$$

In the lab we are interested in the probability by unit of time, that is the transition rate $\Gamma$ :

$$
\Gamma(\alpha \rightarrow \beta)=\frac{(2 \pi)^{D+1}}{\prod_{\alpha} 2 p_{(i)}^{0} V} V \delta^{(D+1)}\left(p_{(\beta)}-p_{(\alpha)}\right)\left|M_{\beta \alpha}\right|^{2} \mathrm{~d} \beta .
$$

In the case where $\alpha$ contains one particle, (1.40) yields decay rates, while for the case in which $\alpha$ contains two particles, it gives cross sections.

\subsection{Unitarity and factorization}

Imagine we are scattering

$$
\alpha=\left|\left\{p_{(i)}, a_{(i)}\right\}\right\rangle \rightarrow \beta=\left|\left\{p_{(j)}, a_{(j)}\right\}\right\rangle .
$$

If the momenta are such that $\left(\sum_{A \subset \alpha} p_{(i)}-\sum_{B \subset \beta} p_{(j)}\right)^{2}=-m^{2}$, where $m$ is the mass of one of the physical 1-particles states of the theory $|p, a\rangle$, then the following process becomes possible:

$$
A \rightarrow B+P \quad, \quad P+\bar{A} \rightarrow \bar{B}, \quad(\bar{A}=\alpha \backslash A, \quad \bar{B}=\alpha \backslash B),
$$

where a subset of the particles, $A$ and $B$, interact first producing an intermediate particle $P=|p, a\rangle$, which then interacts with the others. The likelihood of this event with respect to the particles interacting all at once is infinite.

This singularity is captured by the unitarity properties of the S-matrix, which determine that this singularity is a simple pole, together with its residue. The aim of this section is to prove this result, using only the constructions we have dealt with so far.

Following [1], we do the proof for the particular example of a $3 \rightarrow 3$ scattering ${ }^{3}$. This should illustrate why the singularity is a simple pole, and it will be clear that this carries through to the

\footnotetext{
${ }^{3}$ Notice that in [1] only massive particles were considered. Although it does not the change the structure of the proof, the combinatorics are more tedious when we allow for massless particles. Also here we work in generic $D+1$ dimensions.
} 
general case. Since the proof will be mainly diagrammatic, it is useful to recall our definition (1.29) of the scattering amplitude

$$
\begin{aligned}
& \beta \overline{\vdots(C) \vdots} \alpha=S_{\beta \alpha}^{C}=i(2 \pi)^{D+1} \delta^{(D+1)}\left(p_{(\beta)}-p_{(\alpha)}\right) M_{\beta \alpha}, \\
& \beta \overline{\vdots \quad C^{\dagger} \vdots} \alpha=S_{\alpha \beta}^{\dagger C}=-i(2 \pi)^{D+1} \delta^{(D+1)}\left(p_{(\beta)}-p_{(\alpha)}\right) M_{\beta \alpha}^{\dagger}, \quad M_{\beta \alpha}^{\dagger}=\left(M_{\alpha \beta}\right)^{*} .
\end{aligned}
$$

The connectedness structure of a $3 \rightarrow 3$ scattering process, $P_{1}+P_{2}+P_{3} \rightarrow P_{4}+P_{5}+P_{6}$ is

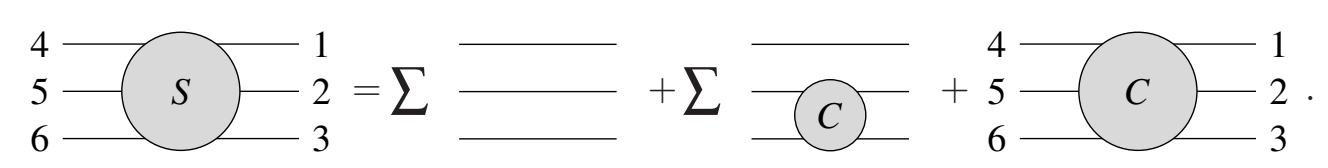

Now let us impose unitarity for this $3 \rightarrow 3$ process:

$$
\left\langle P_{4} P_{5} P_{6} \mid P_{1} P_{2} P_{3}\right\rangle=\left\langle P_{4} P_{5} P_{6}\left|S S^{\dagger}\right| P_{1} P_{2} P_{3}\right\rangle=\int \mathrm{d} \alpha\left\langle P_{4} P_{5} P_{6}|S| \alpha\right\rangle\left\langle\alpha\left|S^{\dagger}\right| P_{1} P_{2} P_{3}\right\rangle .
$$

Diagrammatically, we will express equation (1.44) as

$$
\begin{aligned}
& \sum \bar{\amalg}=\int \mathrm{d} \alpha\left(\sum \bar{\amalg} \vdots \alpha+\sum \overline{\mathrm{C}}: \alpha+\sum \overline{\mathrm{C}} \vdots \alpha\right. \\
& \left.+ 5 \longdiv { 6 } - ( C ): \alpha+\sum \overline{-(C)}: \alpha+\sum \overline{-C E} \cdot \alpha\right) \\
& \left(\sum \alpha \vdots \bar{\square}+\sum \alpha: \overline{\bar{F}}+\sum \alpha \vdots \overline{C^{\dagger}}\right.
\end{aligned}
$$

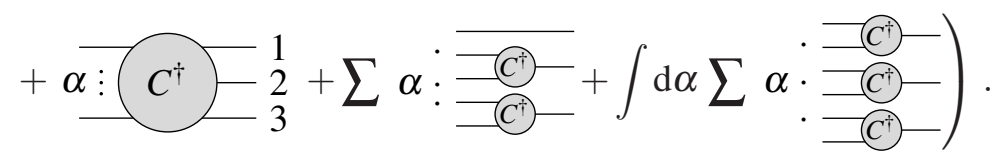

If we expand this expression we will obtain more than the naive $6 \times 6=36$ terms we could expect, since there are several ways of combining the diagrams involving smaller blobs (those with just one or two particles on one side). The way to multiply terms could be defined precisely, but it is the intuitive one. Let us work out a couple of examples to illustrate the procedure:

$$
\begin{aligned}
& \left(\int \mathrm{d} \alpha \underset{6}{5}-(C) \vdots \alpha\right)\left(\sum \alpha \vdots \bar{\square}\right)=\int \mathrm{d} \alpha\left\langle P_{4} P_{5} P_{6}\left|S^{C}\right| \alpha\right\rangle \delta\left(\alpha-P_{1} P_{2} P_{3}\right)
\end{aligned}
$$

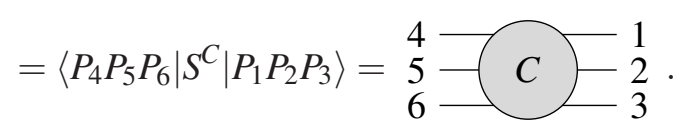

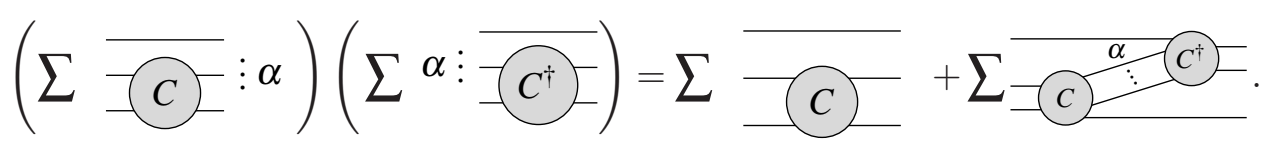

When we look at the terms in (1.45), we notice that not all of them share the same delta-functions. This means that the equation holds in connected components. We focus on the totally connected 
diagrams (this means that no initial particle is equal to a final one). The totally connected part of equation (1.45) reduces to
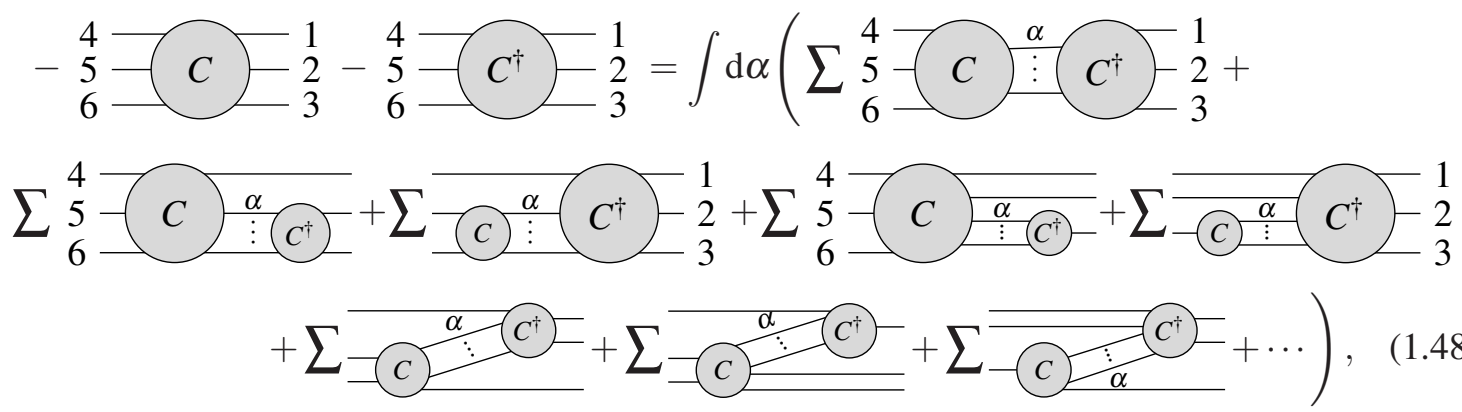

where the dots are standing for terms involving at least three blobs. We only drew the ones with two blobs just to avoid diagrams cluttering.

Equation (1.48) contains all the power of unitarity. On the LHS, we essentially have the imaginary part of the amplitude, while on the RHS we have products of two amplitudes. Obviously, LHS and RHS must be the same. Typically one analyzes the implications of unitarity for the branch cut structure of the amplitude. Performing a perturbative expansion like (1.1), one gets that the discontinuities along branch cuts (i.e. the jump of the imaginary part) of loop-level amplitudes are given by products of amplitudes with less loops, but more legs. Here tough, we will pay attention to a different kind on singularities, different from branch cuts, and where we stay at the same loop order on the LHS and RHS of the unitarity equation, being therefore forced to relate amplitudes to products of other amplitudes with less external legs.

Let us focus on the following particular singularity of the RHS of equation (1.48): when the state $|\alpha\rangle$ is a single-particle state, $P_{a} \equiv|p, a\rangle$ (notice that $\mathrm{d} \alpha \rightarrow \sum_{a}$ ), in the next-to-last diagram of the second line of (1.48), we have a term like

$$
\begin{aligned}
& { }_{6}^{4}=\left(C C_{3}^{P}{ }_{2}^{1}=\frac{1}{(2 \pi)^{D}} \int \frac{\mathrm{d}^{D} \vec{p}}{2 p^{0}} \sum_{a}\left\langle P_{5} P_{6}\left|S^{C}\right| P_{3} P_{a}\right\rangle\left\langle P_{4} P_{a}\left|S^{C \dagger}\right| P_{1} P_{2}\right\rangle\right. \\
& =\frac{1}{(2 \pi)^{D}} \int \mathrm{d}^{D+1} p \delta\left(p^{2}+m^{2}\right) \sum_{a} i(2 \pi)^{D+1} \delta^{D+1}\left(p_{(56)}-p_{(3)}-p\right) M\left(P_{3} P_{a} \rightarrow P_{5} P_{6}\right) \\
& i(2 \pi)^{D+1} \delta^{D+1}\left(p_{(4)}+p-p_{(12)}\right) M^{\dagger}\left(P_{1} P_{2} \rightarrow P_{4} P_{a}\right) \\
& =i(2 \pi)^{D+1} \delta^{(D+1)}\left(p_{(456)}-p_{(123)}\right)(-2 \pi i) \delta\left(k^{2}+m^{2}\right) \sum_{a} M\left(P_{3} P_{a} \rightarrow P_{5} P_{6}\right) M^{\dagger}\left(P_{4} P_{a} \rightarrow P_{1} P_{2}\right) \text {, }
\end{aligned}
$$

where $m$ is the mass of particle $P_{a}$, and we are denoting

$$
p_{\left(i_{1} \ldots i_{m}\right)}=p_{\left(i_{1}\right)}+\ldots+p_{\left(i_{m}\right)}, \quad k=p_{(5)}+p_{(6)}-p_{(3)} .
$$

Now let us pause to observe the singularity structure of the last line of (1.49). Apart from the standard momentum-conservation delta-function, we have the extra $\delta\left(k^{2}+m^{2}\right)$. It is easy to convince oneself that this singularity is not present in any of the other terms on the RHS of (1.48). Therefore, it must be contained on the LHS, which only contains $M\left(P_{1} P_{2} P_{3} \rightarrow P_{4} P_{5} P_{6}\right)$ and $M^{\dagger}\left(P_{1} P_{2} P_{3} \rightarrow\right.$ 
$\left.P_{4} P_{5} P_{6}\right)$. In other words, the amplitude $M\left(P_{1} P_{2} P_{3} \rightarrow P_{4} P_{5} P_{6}\right)$ must contain some singularity that cancels the extra delta-function on the RHS. We want to see that this singularity is a simple pole.

Given the form of the last line of (1.49), to match the singularities with the LHS of (1.48), one is lead to propose:

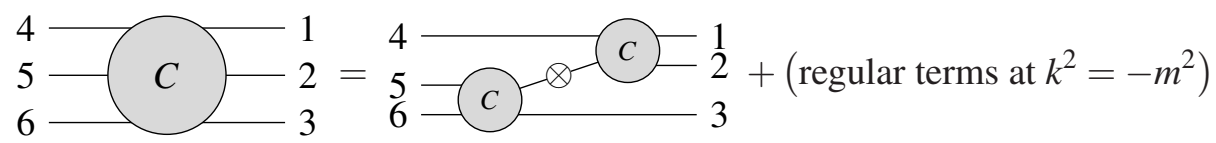

$$
\begin{aligned}
& \equiv i(2 \pi)^{D} \delta^{(D+1)}\left(p_{(456)}-p_{(123)}\right) G\left(k^{2}\right) \sum_{a} M\left(P_{1} P_{2} \rightarrow P_{4} P_{a}\right) M\left(P_{4} P_{a} \rightarrow P_{5} P_{6}\right)+(\text { reg. }),
\end{aligned}
$$

where $G\left(k^{2}\right)$ gives the singular behavior at $k^{2}=-m^{2}$, and in the remaining of the section (reg.) stands for regular terms at $k^{2}=-m^{2}$. It is important to notice that $G$ only depends on $k^{2}$, so this factorization property will hold for any amplitude involving particles $P_{3}, P_{5}$ and $P_{6}$, or (by momentum conservation) $P_{1}, P_{2}$ and $P_{4}$. We have a similar decomposition for $M^{\dagger}$ :

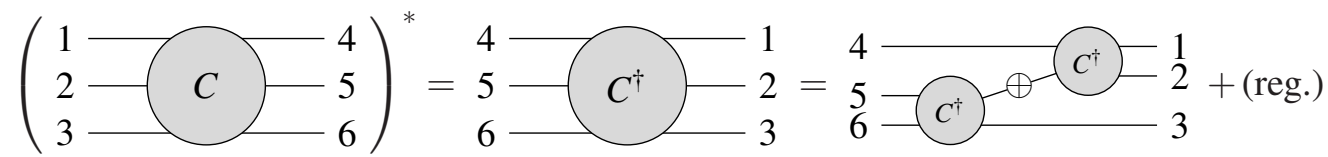

$$
\begin{aligned}
& \equiv-i(2 \pi)^{D} \delta^{(D+1)}\left(p_{(456)}-p_{(123)}\right) G^{\dagger}\left(k^{2}\right) \sum_{a} M^{\dagger}\left(P_{1} P_{2} \rightarrow P_{4} P_{a}\right) M^{\dagger}\left(P_{4} P \rightarrow P_{5} P_{6}\right)+\text { (reg.) },
\end{aligned}
$$

We have to determine $G\left(k^{2}\right)$. In order to do that, let us introduce the ansatz (1.51)-(1.52) in the unitarity equation (1.48) that we want to solve. On the LHS we get minus the following

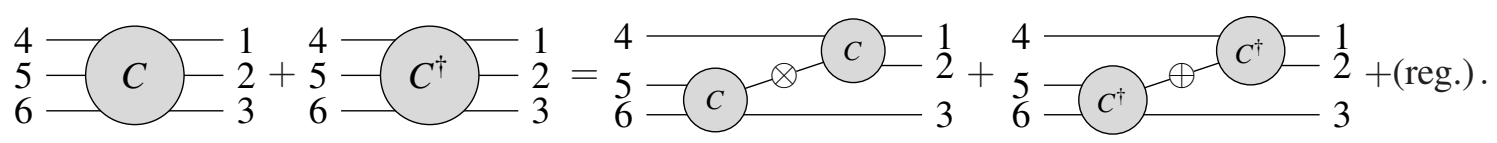

On the RHS we get an obvious contribution from the sixth term inside the integral, and via (1.51)(1.52) we get contributions from the other terms that contain either a blob with particles $P_{3}, P_{5}$ and $P_{6}$ or either with $P_{1}, P_{2}$ and $P_{4}$. So we have for the RHS:

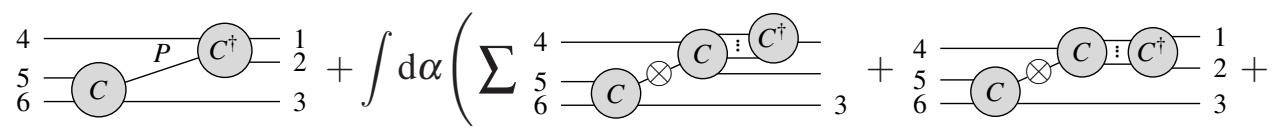

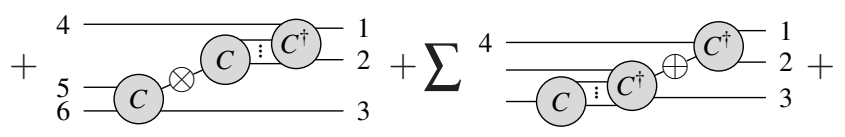

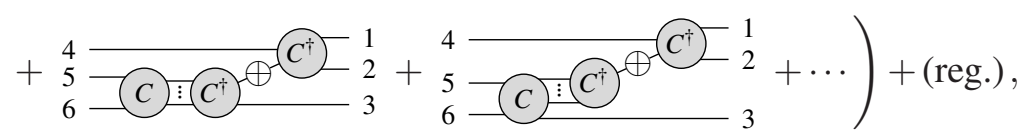

where again the dots contain diagrams with at least four blobs that we are not drawing here. This expression can be simplified if we use the totally connected component of the unitarity equation 
for a $2 \rightarrow 2$ scattering, which can be obtained as we did in (1.48), and reads

$$
\begin{aligned}
& -{ }_{d}^{c} C{ }_{b}^{a}-{ }_{d}^{c} C_{b}^{\dagger}=\int \mathrm{d} \alpha\left(\sum-C C^{\dagger}{ }^{a}\right. \\
& +{ }_{d}^{c} C\left(C^{\dagger}{ }_{b}^{a}+\sum_{d}^{c} C(C)+\cdots\right) .
\end{aligned}
$$

The dots contain once more terms with at least (in this case exactly) three blobs. We can see that the combination of the two blobs in the right-upper corner of the first three terms inside the integral in (1.54) combine precisely in the same way as the terms on the RHS of (1.55). It can be easily, but tediously, checked that the terms we are omitting with the dots combine in this same way. The same happens for the two blobs of the left-lower corner of the last three terms inside the integral of (1.54). We can therefore write for the RHS of (1.48):

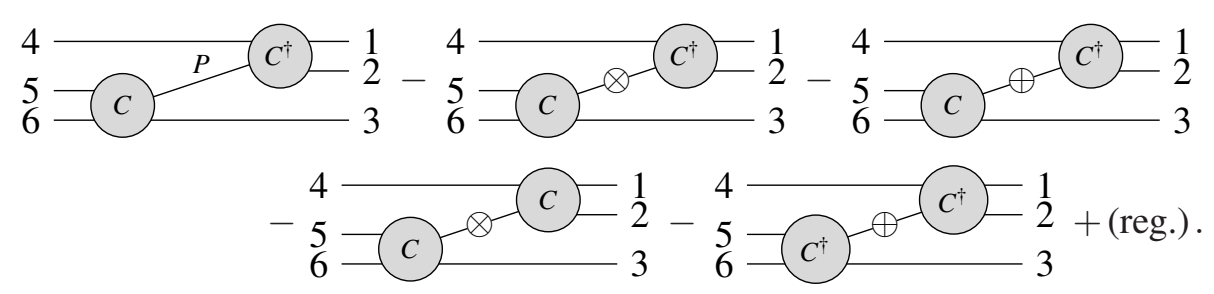

Finally, equating (1.53) and (1.56), we get

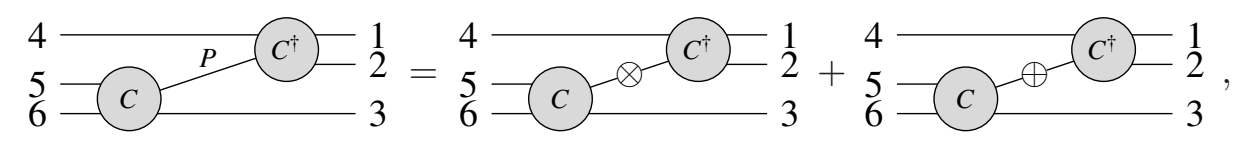

which if we take into account (1.49) and (1.51)-(1.52), translates analytically into

$$
2 \pi i \delta\left(k^{2}+m^{2}\right)=G\left(k^{2}\right)-G^{*}\left(k^{2}\right) .
$$

Recalling how the delta-function arose in (1.49), we have finally arrived to the conclusion that the properties of the singularity are essentially determined by Poincaré invariance. To solve the boxed equation above, we just need to use the well-known ${ }^{4}$ formula

$$
(E \pm i \varepsilon)^{-1}=\frac{\mathscr{P}}{E} \mp i \pi \delta(E) \quad \Longrightarrow \quad \frac{1}{E-i \varepsilon}-\frac{1}{E+i \varepsilon}=2 \pi i \delta(E),
$$

where $\mathscr{P}$ denotes the principal part. Equation (1.58) thus becomes

$$
G\left(k^{2}\right)-G^{*}\left(k^{2}\right)=\frac{1}{k^{2}+m^{2}-i \varepsilon}-\frac{1}{k^{* 2}+m^{2}+i \varepsilon},
$$

whose solution is

$$
G\left(k^{2}\right)=\frac{\lambda}{k^{2}+m^{2}-i \varepsilon}-\frac{1-\lambda}{k^{* 2}+m^{2}+i \varepsilon}, \quad \lambda \in \mathbb{R} .
$$

\footnotetext{
${ }^{4}$ More explicitly, equation (1.59) is just the definition of the principal part: $\int_{-\infty}^{\infty} \mathrm{d} E \frac{f(E)}{E \pm i \varepsilon}=\mathscr{P} \int_{-\infty}^{\infty} \mathrm{d} E \frac{f(E)}{E} \mp i \pi f(0)$ when $f$ is regular at $E=0$.
} 
On the basis of analyticity, we can only have $\lambda=0$ or $\lambda=1$ in (1.61). One can argue [1] that

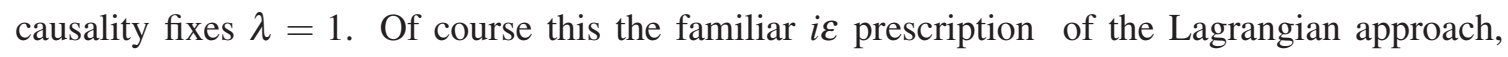
which indeed enforces causality in that context.

Plugging the obtained value for $G\left(k^{2}\right)$ in (1.51), we have arrived to the following factorization property:

$$
M\left(P_{1} P_{2} P_{3} \rightarrow P_{4} P_{5} P_{6}\right)=\sum_{a} M\left(P_{1} P_{2} \rightarrow P_{4} P_{a}\right) \frac{1}{k^{2}+m^{2}-i \varepsilon} M\left(P_{4} P_{a} \rightarrow P_{5} P_{6}\right)+(\text { reg. }) .
$$

There is nothing particular to the $3 \rightarrow 3$ scattering that we have used, so this factorization property holds generally:

$$
M(\alpha \rightarrow \beta)=\sum_{a} M\left(A \rightarrow B+P_{a}\right) \frac{1}{p^{2}+m^{2}-i \varepsilon} M\left(P_{a}+\bar{A} \rightarrow \bar{B}\right)+\left(\begin{array}{c}
\text { regular } \\
\text { terms } \\
\text { at } p^{2}=-m^{2}
\end{array}\right),
$$

where $A$ and $\bar{A}$ are complementary subsets of $\alpha, B$ and $\bar{B}$ are complementary subsets of $\beta, m$ is the mass of particle $P$ and $p=p_{(A)}-p_{(B)}$. One can notice that the amplitudes on the RHS of (1.63) contain less external legs than the one on the LHS. Because of this, we will typically refer to them as sub-amplitudes (which has no particular meaning other than their having a smaller number of external legs; they are perfectly good amplitudes!). Although sometimes equation (1.63) is referred to as the tree-level incarnation of unitarity, notice that it does not refer to any tree-level property of the amplitude; it holds at loop level too!

When the initial kinematical data is such that $p^{2} \simeq-m^{2}$, the amplitude is dominated by the contribution of the pole shown in (1.63). This kinematical limit is called a multi-particle channel. The case in which any of the two sub-amplitudes contains only three particles is a bit special and such a limit is called a collinear limit: conservation of momenta implies that at least two momenta must be collinear, and if one particle is massless the three-point amplitude must vanish because the process is kinematically prohibited. In the former case, instead of a three-point amplitude, one should use a so-called splitting function. This is all assuming real momenta. However, as we will massively exploit later (from Section 2.3 onwards), three-particle amplitudes do exist if we let the momenta to be complex. The steps we have followed to arrive to (1.63) could be performed with external complex momenta too, so the equation has to be valid when the external momenta are allowed into the complex plane.

In conclusion, we have seen how unitarity determines the so-called factorization properties of the amplitude. When several of the scattering particles combine kinematically to allow for the production of another particle, the amplitude is singular, and the singularity is a simple pole in the propagator of this internal particle. Unitarity also determines the residue of the amplitude: it is the product of two amplitudes with fewer external legs.

\subsection{Crossing symmetry}

The existence of antiparticles can be deduced as a means to having conservation of quantum charges. But it can be inferred from an S-matrix perspective too. Let us look at the following pole 
in the scattering amplitude for the process $P_{1}+P_{2}+P_{3}+P_{4} \rightarrow P_{5}+P_{6}+P_{7}+P_{8}$ :
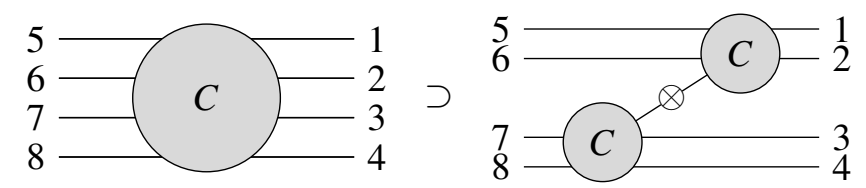

at $k^{2}=-m^{2}$,

where $k=p_{(56)}-p_{(12)}$ and $m$ is the mass of a one-particle state of the theory. We said that we interpreted this pole as the process

$$
P_{1}+P_{2} \rightarrow P_{5}+P_{6}+P \quad ; \quad P+P_{3}+P_{4} \rightarrow P_{7}+P_{8} .
$$

However, for this interpretation to be kinematically possible, it requires, for real momenta,

$$
\sqrt{-p_{(12)}^{2}} \geq \sqrt{-p_{(56)}^{2}}+m, \quad \sqrt{-p_{(78)}^{2}} \geq \sqrt{-p_{(34)}^{2}}+m .
$$

To prove (1.65), one has to use the property

$$
\begin{aligned}
-p \cdot p^{\prime} \gtreqless \sqrt{-p^{2}} \sqrt{-p^{\prime 2}}, & \geq \text { if } \operatorname{sign} p^{0}=\operatorname{sign} p^{\prime 0} \\
& \leq \text { if } \operatorname{sign} p^{0} \neq \operatorname{sign} p^{\prime 0}
\end{aligned},
$$

combined $^{5}$ with the fact that $p_{(12)}^{0}, p_{(56)}^{0}, k^{0}>0$. But, on the physical region $\left(p_{(i)}\right.$ real, $p_{(i)}^{0}>0$, $p_{(i)}^{2}=-m_{(i)}^{2}$ ), nothing prevents that the kinematical data is such that the opposite condition to (1.65) is satisfied, namely:

$$
\sqrt{-p_{(12)}^{2}} \leq \sqrt{-p_{(56)}^{2}}-m, \quad \sqrt{-p_{(12)}^{2}} \leq \sqrt{-p_{(56)}^{2}}-m .
$$

Then, the pole should be interpreted instead as the process

$$
\begin{array}{ccc}
P_{1}+P_{2}+\bar{P} & \rightarrow P_{5}+P_{6} \\
P_{3}+P_{4} & \rightarrow \bar{P}+P_{7}+P_{8}
\end{array}{ }_{7}
$$

Here $\bar{P}$ has mass $\bar{k}^{2}=-m^{2}$ (where $\bar{k}=-k$ ), the same as $P$, but the quantum numbers should be opposite to those of $P . \bar{P}$ is the antiparticle of $P$ !

It should be clarified that the two processes (1.64), (1.68) cannot happen at the same time. For given kinematical data, we either have $k^{0}>0$ and $\bar{k}^{0}<0$ or vice-versa; either way, in one of the processes we are not in the physical region. This does not pose a problem since we are assuming analyticity and we are free to analytically continue the scattering amplitude.

The factorization of the amplitude $M\left(P_{1} P_{2} P_{3} P_{4} \rightarrow P_{5} P_{6} P_{7} P_{8}\right)$ is uniquely given by (1.63). Since we have the two possible interpretations (1.64) and (1.68) for the resulting pole, we must have

$$
M\left(P_{1} P_{2} \rightarrow P P_{3} P_{4}\right) M\left(P P_{3} P_{4} \rightarrow P_{7} P_{8}\right)=M\left(P_{1} P_{2} \bar{P} \rightarrow P_{5} P_{6}\right) M\left(P_{3} P_{4} \rightarrow \bar{P} P_{7} P_{8}\right) .
$$

\footnotetext{
${ }^{5}$ More detailedly: $m^{2}=-\left(p_{(56)}-p_{(12)}\right)^{2}=-p_{(12)}^{2}-p_{(56)}^{2}+2 p_{(12)} \cdot p_{(56)} \leq\left(\sqrt{-p_{(12)}^{2}}-\sqrt{-p_{(56)}^{2}}\right)^{2}$. Writing $p_{(12)}=$ $p_{(56)}+k$, it follows that $-p_{(12)}^{2}=-p_{(56)}^{2}+m^{2}-2 p_{(56)} \cdot p \geq-p_{(56)}^{2}+m^{2}+2 m \sqrt{-p_{(56)}^{2}} \geq-p_{(56)}^{2}$ since $-p_{(56)}^{2}>0$.
} 
Since this equation holds for any process we want, we must conclude that

$$
M(\ldots P \rightarrow \ldots)=\zeta M(\ldots \rightarrow \bar{P} \ldots) \quad \text { and } \quad M(\ldots \rightarrow P \ldots)=\zeta^{-1} M(\ldots \bar{P} \rightarrow \ldots)
$$

We have not discussed a property called Hermitian analyticity. It states that the matrix elements of $S$ and $S^{\dagger}$ are conjugate to each other in the real sheet. This property can be derived from our postulates too (see chapter 4.6 of [1]). Using Hermitian analyticity, it can be proved that $|\zeta|=1$. By choosing appropriately the phase of the one-state vectors (notice that so far $|p, a\rangle$ is defined up to a phase), we can choose $\zeta=1$. Then we have

$$
M(\alpha \rightarrow \beta)=M(\alpha+\bar{P} \rightarrow \beta-P)=M(\alpha-P \rightarrow \beta+\bar{P})
$$

This property is called crossing symmetry.

From now on, using crossing symmetry, we consider processes where all the particles are ingoing (see figure 1). In this way, all particles are on an equal footing. Notice that the factorization

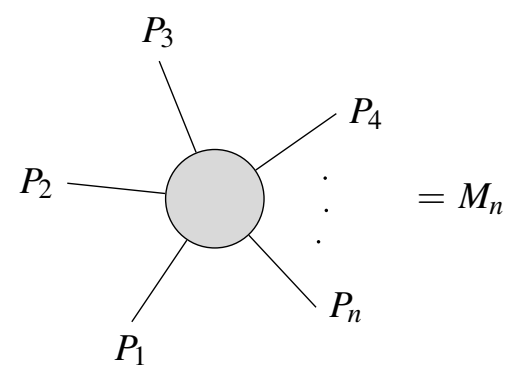

Figure 1: Representation of the amplitude $M\left(P_{1}+\ldots+P_{n} \rightarrow \varnothing\right)=M_{n}$. Using crossing symmetry, we will consider scattering processes where all the particles are incoming.

of scattering amplitudes on multi-particle channels/collinear limits, equation (1.63), is re-expressed as

$$
M_{n}(\alpha) \rightarrow \sum_{a} M_{n-k+1}\left(A+P_{a}\right) \frac{1}{p_{(A)}^{2}+m^{2}} M_{k+1}\left(\bar{A}+\bar{P}_{\bar{a}}\right)
$$

where $\bar{P}_{\bar{a}}$ is the antiparticle of $P_{a}$. Recall that $p_{(A)}=\sum_{i \in A} p_{(i)}$, and in the multi-particle channel $p_{(A)}^{2}=-m^{2}$, where $m$ is the mass of particle $P$. We are omitting the $i \varepsilon$ because we will not need it for the rest of the lectures. Equation (1.72) is the version of unitarity we will use all along Section 2 .

\subsection{Soft limits}

There is a particular class of collinear limit that occurs in theories that contain massless particles in their spectrum. It was first studied by Weinberg in the classical paper [7]. Let us consider a process $\alpha+P \rightarrow \varnothing$, where we let the momentum $p$ of the massless particle $P$ go to zero. Since $\left(p_{(j)}+p\right)^{2}=p_{(j)}^{2}+2 p \cdot p_{(j)} \rightarrow-m_{(j)}^{2}$ for any $j$, because of factorization (1.72) the amplitude must 
show a pole in this limit, that is called a soft limit:

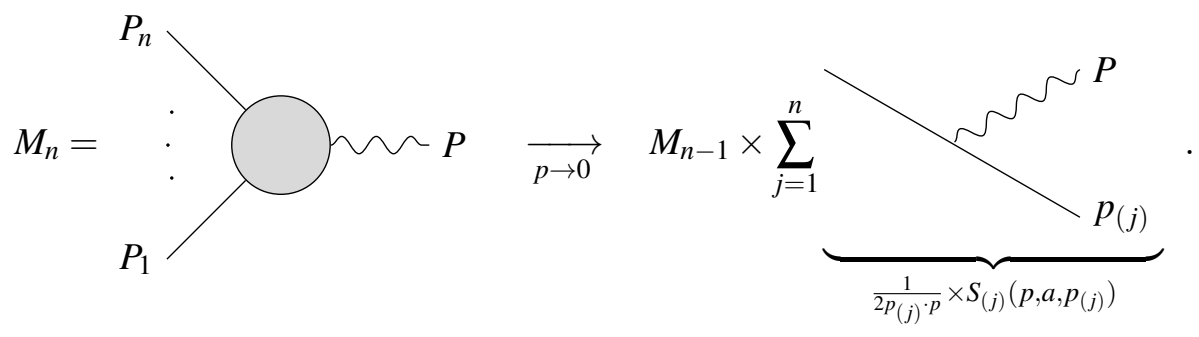

Notice that the factorization can only occur if $P$ is a particle of integer helicity. The object on the right of equation (1.73), $S_{(j)}$ is not exactly an amplitude because it is only defined for $p=0$. It is called a soft factor. It is tempting to think of it as the limit of a three-point amplitude as we take one of the participating momenta to zero, but that gives a singular object. Moreover, one cannot really distinguish between the emission of one soft particle and, say, two soft particles. These IR divergencies could change the structure of the factorization (1.73).

We will stay at the perturbative level, safe from these considerations ${ }^{6}$. Then the soft factors $S_{(j)}$ can be determined only from their Lorentz tensor properties. We refer the reader for the detailed derivation to the original Weinberg paper [7], or to some of the many papers where his argument has been revisited, like for instance the recent $[6,9]$. Yet, let us comment on the interesting conclusions that can be extracted from the soft limits. In four dimensions we can write the compact formula:

$$
S_{(j)}\left(p, \pm h, p_{(j)}\right)=g_{(j)}\left[\varepsilon_{ \pm}(p) \cdot p_{(j)}\right]^{h},
$$

where $h$ is the (absolute value of the) helicity of the soft particle $P$, and $\varepsilon$ is a polarization vector, to be introduced later in Section 2. The constant $g_{(j)}$ can be identified with the strength of the interaction. Indeed (1.74) is a dynamical definition of the coupling constant for long-range interactions. Specifying for photons (i.e. massless spin-1 particles) and gravitons (massless spin-2 particles), the soft limits are:

$$
\begin{array}{cl}
\text { For photons: } \quad M_{n+1} \rightarrow \sum_{j=1}^{n} g_{(j)} \frac{\varepsilon_{ \pm}(p) \cdot p_{(j)}}{p \cdot p_{(j)}} M_{n} . \\
\text { For gravitons: } \quad M_{n+1} \rightarrow \sum_{j=1}^{n} g_{(j)} \frac{\left(\varepsilon_{ \pm}(p) \cdot p_{(j)}\right)^{2}}{p \cdot p_{(j)}} g_{(j)} M_{n} .
\end{array}
$$

By requiring that the factorized expression (1.73) has the right Lorentz covariance properties (1.31), in [7] Weinberg proved that the only consistent possibilities are

- $h=0$, which corresponds to soft massless scalars.

- $h=1$ if one imposes $\sum_{j} g_{(j)}=0$, which is equivalent to the conservation of (a dynamically defined) charge.

- $h=2$ if one imposes $g_{(i)}=g_{(j)}$, which is the quantum formulation of the equivalence principle: all matter has the same gravitational charge.

\footnotetext{
${ }^{6}$ Weinberg studied these IR divergencies in [8]. He concluded that the factorization (1.73) together with (1.76) is true non-perturbatively too!
} 
The soft factors (1.74) for particles with $h \geq 3$ are not consistent with the transformation rules (1.31). So for this type of high-spin particles, it seems Weinberg's result says that no consistent unitary and Lorentz-covariant S-matrix can be constructed. But this is not exactly true. What the result is saying is that we must have $g_{(j)}=0$ in (1.74) for $h \geq 3$. This means that high-spin particles cannot interact at zero frequency, or equivalently, that they cannot produce macroscopic effects.

Of course this statement holds as long as one keeps the assumptions we have made. A particular way out is to consider the existence of an infinite tower of high-spin particles, as this would spoil the factorization (1.72) since the singularity structure could be different, given that there is an infinite number of particles that can propagate internally. A similar loophole can be found if we consider irreducible representations of the Lorentz group different than the standard helicity ones we are considering, which also translates into a different singularity structure (an essential singularity instead of a simple pole; see [9] for a detailed study).

\subsection{Reconstructing the amplitude at tree level}

To the extent to which we want to treat the subject of the S-matrix program, we are finished with the formal properties of the amplitude. We can now start to think about how can we reconstruct the amplitude from its singularity structure. We will focus on the tree-level part of the amplitude (recall this is defined by (1.1)). The reason is two-fold: the tools we have reviewed are not enough to study the loop level, and more importantly, at present we do not know yet how to tackle the loop level in a generic fashion.

From unitarity, we have seen that the only singularities of tree-level scattering amplitudes are factorization channels and soft limits, and these singularities are simple poles (in the invariant squared momenta variables). The tree-level amplitude is therefore a meromorphic function. An honest question we can ask is: when do the locations of these poles plus their residues (i.e. physical information) determine the amplitude? Another way to formulate the question is: if we build an object whose only singularities, and corresponding residues, correspond with the expected factorization channels and soft limits of a tree-level amplitude (which are known beforehand); is it the actual amplitude?

It is believed that this is the case, but as far as I know there is no proof of this fact. In the case of one variable $z$, and with certain assumptions, the answer is affirmative. For a rational function, the knowledge of its poles together with its residues, and the location of some zeroes in the case the function does not vanish at $z=\infty$, is enough, and we can reconstruct the function. Of course the trick is how to map a problem of multi-variable complex calculus (the amplitude $M_{n}$ is a meromorphic function of $n(D+1)$ variables) into a problem with a single complex variable. This trick was invented in [10] by Britto, Cachazo, Feng and Witten (BCFW), and it will our concern for almost the whole of the next section.

\section{Building tree-level amplitudes without Lagrangians}

From now on, we restrict ourselves to tree-level scattering. We will omit then the label 'tree' in our object of study, the tree-level scattering amplitude:

$$
M\left(\left\{p_{(i)}, a_{(i)}, \Upsilon_{(i)}\right\}\right) \text { for the process } P_{1}+\ldots+P_{n} \rightarrow \varnothing,
$$


where we separate the little group indices $a_{(i)}$ from the other quantum labels $\Upsilon_{(i)}$ of the particles. As we have discussed, tree level means that the only singularities of $M$ are the simple poles arising from factorization channels and soft limits. $M$ is in principle a meromorphic function; although later we will be more restrictive and declare it to be rational.

Before getting more serious about the computation of $M$ in (2.1), let us take a couple of minutes to convince ourselves that this is the object we should be studying, at least in the case we only have massless particles. To best appreciate the Lorentz structure of a scattering amplitude, we recast its transformation properties (1.31) as

$$
M_{a_{(1)} \ldots a_{(n)}}\left(\left\{p_{(i)}, \Upsilon_{(i)}\right\}\right)=D\left(\Lambda, p_{(1)}\right)_{a_{(1)} b_{(1)}} \cdots D\left(\Lambda, p_{(n)}\right)_{a_{(n)} b_{(n)}} M_{b_{(1)} \ldots b_{(n)}}\left(\left\{\Lambda p_{(i)}, \Upsilon_{(i)}\right\}\right)
$$

where the sum over the indices $b_{(i)}$ on the RHS is implicit. Since the quantum numbers $\Upsilon_{(i)}$ are not affected by Lorentz transformations, from here on we will not write them explicitly. Recalling that the $D(\Lambda)_{a b}$ 's furnish a representation of the little group, we find then that the scattering amplitude transforms with little group indices. However, in the literature it is more common to find an object that transforms as a Lorentz tensor, especially because this is what we get directly from Feynman diagrams ${ }^{7}$. Such an object is called a "tensor amplitude". How can we build it from the amplitude $M$ in (2.1)? Let us discuss the case where we have only massless bosons.

The way to write a tensor amplitude from our $M$ is via the so-called polarization "tensors", objects which translate from little group indices to Lorentz ones. The explicit passage for their construction in $D=3$ can be found in [7], and it can be generalized to arbitrary $D$. Calling $s_{(i)}$ the "spin" of particle $i$, its polarization "tensor" is $e_{a_{(i)}}^{\mu_{1}^{(i)} \ldots \mu_{s(i)}^{(i)}}\left(p_{(i)}\right)$ such that:

$$
M_{a_{(1)} \ldots a_{(n)}}=e_{a_{(1)}}^{\mu_{1}^{(1)} \ldots \mu_{s(1)}^{(1)}}\left(p_{(1)}\right) \ldots e_{a_{(n)}}^{\mu_{1}^{(n)} \ldots \mu_{s(n)}^{(n)}}\left(p_{(n)}\right) M_{\mu_{1}^{(1)} \ldots \mu_{s(1)}^{(1)} \ldots \mu_{1}^{(n)} \ldots \mu_{s(n)}^{(n)}},
$$

with the tensor amplitude verifying the transformation property:

$$
\begin{aligned}
& M_{\mu_{1}^{(1)} \ldots \mu_{s(1)}^{(1)} \ldots \mu_{1}^{(n)} \ldots \mu_{s(n)}^{(n)}}\left(\left\{p_{(i)}\right\}\right)= \\
& \Lambda_{\mu_{1}^{(1)}}^{v_{1}^{(1)}} \cdots \Lambda_{\mu_{s_{(1)}}^{(1)}}^{v_{s_{(1)}}^{(1)}} \cdots \Lambda_{\mu_{n}^{(1)}}^{v_{n}^{(1)}} \cdots \Lambda_{\mu_{s(n)}^{(n)}}^{v_{s(n)}^{(n)}} M_{v_{1}^{(1)} \ldots v_{s_{(1)}}^{(1)} \cdots v_{1}^{(n)} \ldots v_{s_{(n)}}^{(n)}}\left(\left\{\Lambda p_{(i)}\right\}\right) .
\end{aligned}
$$

The transformation properties (1.31) of the amplitude $M$ together with the requirement (2.4) determine (not completely) the form of the polarization "tensors" and their Lorentz properties. In four dimensions, it turns out that they can be built out of polarization "vectors" $\varepsilon_{a}^{\mu}(a= \pm)$ :

$$
e_{a}^{\mu_{1} \ldots \mu_{s}}(p)=\varepsilon_{a}^{\mu_{1}} \cdots \varepsilon_{a}^{\mu_{s}}(p)
$$

which are just polarization "tensors" for particles of spin 1 . The quotation marks, that we will drop from now on, are there to remind us that nor $e_{a}^{\mu_{1} \ldots \mu_{s}}$ is a tensor neither $\varepsilon_{a}^{\mu}$ is a vector. They transform non-homogeneously with a Lorentz transformation. They verify the following properties:

$$
\varepsilon_{a}^{\mu} \varepsilon_{a, \mu}^{*}=1, \quad \varepsilon_{a}^{\mu} \varepsilon_{a, \mu}=0, \quad p_{\mu} \varepsilon_{a}^{\mu}(p)=0 .
$$

\footnotetext{
${ }^{7}$ The way to get the S-matrix elements there is by applying the LSZ reduction formula on (Fourier-transforms of) expectation values of time-ordered products of field operators, which naturally come with a Lorentz structure.
} 
It is always possible to find such polarization vectors, but they are defined up to a shift proportional to the momentum (this is linked with their inhomogeneous transformation properties):

$$
\varepsilon_{a}^{\mu}(p) \rightarrow \varepsilon_{a}^{\mu}(p)+\omega p, \quad \omega \in \mathbb{R} .
$$

This can be recognized as a gauge transformation. It was already proven by Weinberg [7] that in order for the S-matrix to transform covariantly (as in (1.23)), we have to require that the tensor amplitudes verify

$$
p_{(i)}^{\mu_{(i)}} M_{\mu_{(1)} \ldots \mu_{(n)}}\left(\left\{p_{(j)}\right\}\right)=0 \quad \forall i .
$$

In virtue of (2.3), we see that the condition above amounts to say that $M_{a_{(1)} \ldots a_{(n)}}$ does not change under (2.7). Or in other words, since (2.7) was identified as a gauge transformation, $M_{a_{(1)} \ldots a_{(n)}}$ is a gauge-invariant object.

Presented in this way, we clearly see that introducing the tensor structure via "polarization tensors" only complicates things. Indeed, the notion of gauge invariance arises only because of the redundancy (2.7) in the construction of tensor amplitudes. Had we not introduced the latter, there would be no need of discussing any redundancy in the description of the physics of a scattering process. Thus, from this point of view $M_{a_{(1)} \ldots a_{(m)}}$ is the natural (gauge-invariant) object to study, at least in the case of massless bosons. Actually it is not hard to extend the construction above to accommodate for massless fermions (one just needs to include a spinor into the game, which tensored with the polarization tensors would yield polarization "spinors"). The case of massive particles would be slightly different, while (2.3)-(2.4) still hold, the construction of polarization tensors would be different owing to the the different structure of the Little Group.

After this small detour, we come back to our main purpose: determining the amplitude at tree level from its singularity structure. As we have said the only singularities at this level of perturbation theory are soft and multi-particle/collinear limits. Can we use this (or just part of this) information to reconstruct the amplitude? More precisely, under soft and multi-particle/collinear limits, the tree-level amplitude factorizes into smaller tree-level amplitudes. The question is whether we can use these smaller pieces to assemble the bigger amplitude. The answer is yes.

- The most explored avenue is the reconstruction of the tree-level scattering amplitude from factorization channels. The archetypical example is the BCFW recursion relation, discovered by just Britto, Cachazo and Feng while studying one-loop QCD amplitudes [11], and later shown (with the addition of Witten to the team) to be just a tree-level property [10]. Actually the first example of reconstruction of amplitudes from factorization channels in this context came a bit before, when Cachazo, Svrcek and Witten discovered how to build generic YangMills amplitudes from just maximally-helicity-violating ones, with the so-called CSW rules [12]. Later it was shown by Risager that the CSW rules can be derived in a similar manner as the BCFW recursion relations, by using a different complex deformation [13]. Actually, the idea of changing the deformation sources many different recursion relations, among which we can mention the all-line shift [14].

- Reconstructing the amplitude from soft limits, on the other hand, has not attracted too much attention despite the appeal of generating $(n+1)$-point amplitudes by attaching an "inverse soft factor" to an $n$-point amplitude. This goal has only been achieved for $\mathscr{N}=4$ SYM [15] 
(see also [16], where the inverse soft operation is stated in terms of on-shell diagrams, and it is seen to be extensible also for loop amplitudes, but only with less than 14 external legs). For other theories, only some partial results have been obtained (see [17] for the study of the "inverse soft paradigm" for graviton and gluon amplitudes), although it has inspired some other riveting developments for graviton amplitudes [18].

- One could also think of a combined approach, where the factorization properties on both soft and multi-particle/collinear limits are used to reconstruct the amplitude. Again this is not a much investigated avenue, but interesting results are available for the scattering of gluons and gravitons [19].

Among all these methods, in what follows we focus on the best studied case, which is also the one that reconstructs the amplitude with the least amount of information: the BCFW recursion relation.

\subsection{BCFW and generalizations}

As we have just mentioned, the $\mathrm{BCFW}$ recursion relation was born as just the $\mathrm{BCJ}$ recursion relation. Britto, Cachazo and Feng came across it while studying the IR behavior of one-loop YM amplitudes [11]. A bit later, together with Witten, they realized that it was not needed to use any one-loop structure, and that the relations were a tree-level property of gluon amplitudes [10]. Actually, their method for deriving the relations is completely generic and works for any theory with massless particles (actually massive particles can be very easily accommodated [20]), if it were not for a certain "boundary term" that sometimes must be included in the recursion relation (BCFW showed that this term could be made to vanish in the YM theory). To give more substance to this story, let us present a derivation of the BCFW recursion relation which departs a bit from its historical roots, but is more in line with the approach we advocate in this lectures and will follow naturally from the results presented in Section 1.

Recall we are studying the scattering process (2.1). Pick up two of the scattering particles, say $P_{i}$ and $P_{j}$ and deform their momenta (this is called a BCFW deformation or BCFW shift) as:

$$
p_{(i)} \rightarrow p_{(i)}(z)=p_{(i)}-z q, \quad p_{(j)} \rightarrow p_{(j)}(z)=p_{(j)}+z q,
$$

where $z \in \mathbb{C}$ and $q$ is a momentum to be determined below. The momenta of the other particles is left untouched. We want to use the analytic properties of the S-matrix, so in order to be able to still talk about a scattering process we need two conditions to be satisfied by the deformation (2.9). One is momentum conservation, which is clearly preserved. The other is that the momenta remain on-shell: $p_{(i)}(z)^{2}=-m_{(i)}^{2}, p_{(j)}(z)^{2}=-m_{(j)}^{2}$. Since the original momenta were on the mass-shell, this condition amounts to requiring

$$
z^{2} q^{2}-2 z q \cdot p_{(i)}=0, \quad z^{2} q^{2}+2 z q \cdot p_{(j)}=0 .
$$

In order to satisfy this equation for any $z$, we have to find $q$ such that

$$
q^{2}=0, \quad q \cdot p_{(i)}=0=q \cdot p_{(j)} .
$$

It is only possible to satisfy (2.11) for $D \geq 3$ and complex $q$. 
Let us suppose then that we have found a $q$ such that the BCFW deformation (2.9) does not take away the interpretation of a scattering process. The only thing that has changed is that now a pair of particles have complex momenta (which does not pose a problem given the analyticity properties of the S-matrix). We can still consider the on-shell amplitude

$$
M_{n} \rightarrow M_{n}\left(\left\{p_{(k)}(z), a_{(k)}\right\}\right) \equiv M_{n}^{(i, j)}(z)
$$

where we are taking into account that $p_{(k)}(z)=p_{(k)}$ if $k \neq i, j$. Now, the key observation is that, as a function of $z, M_{n}^{(i, j)}(z)$ is a rational function with simple poles ${ }^{8}$. This is because, as we have discussed in the first part of the lectures, the only singularities of the S-matrix at tree level are:

Factorization channels They happen whenever the momenta flowing through a certain channel becomes on-shell. In the notation we have been using this condition is expressed as: $p_{(\mathscr{K})}(z)^{2}=-m^{2}$ for a given channel $\mathscr{K}$ and any of the physical masses $m$ of the theory. Recall that $p_{(\mathscr{K})}(z)=\sum_{k \in \mathscr{K}} p_{(k)}$. From the perspective of the $z$-variable, this on-shell condition can be realized whenever either particle $i$ or particle $j$ are in $\mathscr{K}$, but not both. In the latter case, $p_{(\mathscr{K})}(z)$ does not depend on $z$ and it will not be on-shell for generic external kinematical data. In the former case, it is easy to solve for $z$ :

$$
\begin{aligned}
p_{(\mathscr{K})}(z)^{2}+m^{2} & =\left(p_{(\mathscr{K})} \pm z q\right)^{2}+m^{2}=p_{(\mathscr{K})}^{2}+m^{2} \pm 2 z p_{(\mathscr{K})} \cdot q \\
& =\left(p_{(\mathscr{K})}^{2}+m^{2}\right)\left(1-\frac{z}{z_{\mathscr{K}}}\right) \text { defining } z_{\mathscr{K}} \equiv \frac{p_{(\mathscr{K})}^{2}+m^{2}}{\mp 2 p_{(\mathscr{K})} \cdot q} .
\end{aligned}
$$

In view of the equation above and the fact that the factorization (1.72) includes a denominator with the propagator squared, it is clear that $M_{n}^{(i, j)}(z)$ will have simple poles at the locations $z_{\mathscr{K}}$. Although we do not indicate it explicitly, these obviously depend on the particles $(i, j)$ chosen for the deformation.

Soft limits They happen if we have massless particles in the scattering, and the momentum of one of them goes to zero. Except for very particular kinematical configurations, it is not possible to attain $p_{(i)}, p_{(j)} \rightarrow 0$ with the deformation (2.9). So these singularities are invisible to the BCFW deformation.

Therefore, the only singular points of $M_{n}^{(i, j)}(z)$ are simple poles. Moreover we know what the residues at these poles are in virtue of unitarity (1.72):

$$
\begin{aligned}
M_{n}^{(i, j)}(z) \rightarrow \sum_{I} M^{(i, j)}\left(\mathscr{K}\left(z_{\mathscr{K}}\right),-I\left(z_{\mathscr{K}}\right)\right) \frac{1}{p_{(\mathscr{K})}(z)^{2}+m^{2}} M^{(i, j)}\left(\overline{\mathscr{K}}\left(z_{\mathscr{K}}\right), \bar{I}\left(z_{\mathscr{K}}\right)\right) \\
=\sum_{I} \frac{M_{L(\mathscr{K})}^{(i, j)} M_{R(\mathscr{K})}^{(i, j)}}{\left(p_{(\mathscr{K})}^{2}+m^{2}\right)\left(1-\frac{z_{\mathscr{K}}+\varepsilon}{z_{\mathscr{K}}}\right)}=\underbrace{-\sum_{I} \frac{M_{L(\mathscr{K})}^{(i, j)} M_{R(\mathscr{K})}^{(i, j)}}{p_{(\mathscr{K})}^{2}+m^{2}} z_{\mathscr{K}}}_{\text {residue }} \frac{1}{\varepsilon} \quad \text { when } \quad z \rightarrow z_{\mathscr{K}}+\varepsilon,
\end{aligned}
$$

\footnotetext{
${ }^{8}$ Recall the assumptions that play a role here. Essentially we have unitarity, analyticity and 1-particle states that are irreducible representations of the Poincare group. The condition that seems more amenable to a change is the last one. Indeed, it has been argued [9] that if one allows for CSP particles, the S-matrix would contain essential singularities. Having an infinite number of particles that could propagate in a factorization channel would presumably change the singularity structure too.
} 
where $\sum_{I}$ indicates that we have to sum over all possible internal particles $I$ that can propagate inside the channel. That is a sum over little group indices and particle species. The intermediate 1-particle states are $-I\left(z_{\mathscr{K}}\right) \equiv\left|-p_{(\mathscr{K})}\left(z_{\mathscr{K}}\right), a\right\rangle$ and its anti-particle (with opposed momentum) $\bar{I}\left(z_{\mathscr{K}}\right) \equiv\left|p_{(\mathscr{K})}\left(z_{\mathscr{K}}\right), \bar{a}\right\rangle$.

The question is then how do we reconstruct a rational function with prescribed poles and residues. Unless the function vanishes at infinity, there is not a unique answer. Such an answer can be given if we know the value of the amplitude at some set of $z$ 's. A particular set of interesting points seem to be those where the amplitude vanishes ${ }^{9}$; or in other words, the particles do not scatter. How many of them do we need to reconstruct the amplitude? Let us follow the next derivation:

Since the amplitude is a rational function, there must be an integer number $v$ such that

$$
M_{n}^{(i, j)}(z) \sim z^{v} \text { when } z \rightarrow \infty
$$

The exponent $v$ depends on the choice of particles $(i, j)$ (as well as on the choice of $q$ ) for the deformation. When a deformation generates an amplitude that vanishes at infinity, we talk about a good/right BCFW shift; and otherwise, if $v \geq 0$, we say we have performed a bad/wrong BCFW shift.

We can always ${ }^{10}$ write the amplitude as

$$
M_{n}^{(i, j)}(z)=\underbrace{\prod_{l=1}^{v+1}\left(1-\frac{z}{z_{0}^{(l)}}\right)}_{\text {if } v \geq 0} \sum_{\mathscr{K}} \frac{c_{\mathscr{K}}}{1-\frac{z}{z_{\mathscr{K}}}}
$$

as this is the most general expression for a function with the properties we want. The first piece is obviously not present in the case $v<0$. The points $z_{0}^{(l)}$ are (possibly a subset of the set of) zeroes of the amplitude, and $c_{\mathscr{K}}$ are complex numbers to be determined. To do that, we go close to the poles and exploit the factorization properties of the amplitude. On the one hand we have

$$
M_{n}^{(i, j)}\left(z_{\mathscr{K}}+\varepsilon\right) \sim-\prod_{l=1}^{v+1}\left(1-\frac{z_{\mathscr{K}}}{z_{0}^{(l)}}\right) c_{\mathscr{K}} \frac{z_{\mathscr{K}}}{\varepsilon} .
$$

And on the other one we have the factorization (2.14),

$$
M_{n}^{(i, j)}\left(z_{\mathscr{K}}+\varepsilon\right) \sim-\sum_{I} M_{L}^{(i, j)}\left(z_{\mathscr{K}}\right) \frac{1}{p_{(\mathscr{K})}^{2}+m^{2}} M_{R}^{(i, j)}\left(z_{\mathscr{K}}\right) \frac{z_{\mathscr{K}}}{\varepsilon} .
$$

The comparison of equations (2.17) and (2.18) yields

$$
c_{\mathscr{K}}=\sum_{I} M_{L}^{(i, j)}\left(z_{\mathscr{K}}\right) \frac{1}{p_{(\mathscr{K})}^{2}+m^{2}} M_{R}^{(i, j)}\left(z_{\mathscr{K}}\right) \prod_{l=1}^{v+1}\left(1-\frac{z_{\mathscr{K}}}{z_{0}^{(l)}}\right)^{-1} .
$$

\footnotetext{
${ }^{9}$ From the mathematical point of view, also zeroes are particular points. However if for same reason we happened to know the value of the amplitude at certain particular points, we could use them instead of the zeroes [21].

${ }^{10}$ Actually we need to assume that $\sum_{\mathscr{K}} c_{\mathscr{K}} z_{\mathscr{K}} \neq 0$ so that the $M_{n}^{(i, j)}(z)$ in in (2.16) behaves as $z^{v}$ as $z \rightarrow \infty$. This condition is satisfied by all the theories I am aware of.
} 
To give a more physical meaning to the last piece on the RHS above, we use the following simple algebraic identity:

$$
\frac{1}{1-\frac{z_{\mathscr{K}}}{z_{0}^{(l)}}}=1-\frac{1}{1-\frac{z_{0}^{(l)}}{z_{\mathscr{K}}}}=1-\frac{p_{(\mathscr{K})}^{2}+m^{2}}{p_{(\mathscr{K})}^{2}\left(z_{0}^{(l)}\right)+m^{2}}
$$

where in the last step we have used the form of the deformation $p_{(\mathscr{K})}(z)^{2}=p_{(\mathscr{K})}^{2}\left(1-\frac{z}{z_{\mathscr{K}}}\right)$. Thus, we can rewrite (2.19) as

$$
c_{\mathscr{K}}=\sum_{I} M_{L}^{(i, j)}\left(z_{\mathscr{K}}\right) \frac{1}{p_{(\mathscr{K})}^{2}} M_{R}^{(i, j)}\left(z_{\mathscr{K}}\right) \prod_{l=1}^{v+1}\left(1-\frac{p_{(\mathscr{K})}^{2}+m^{2}}{p_{(\mathscr{K})}^{2}\left(z_{0}^{(l)}\right)+m^{2}}\right) .
$$

Plugging this value of $c_{\mathscr{K}}$ in (2.16), and evaluating the expression at $z=0$ we arrive at the following expression for the amplitude:

$$
M_{n}=\sum_{\mathscr{K}} \sum_{I} M_{L}^{(i, j)}\left(z_{\mathscr{K}}\right) \frac{f_{\mathscr{K}}^{(i, j)}}{p_{(\mathscr{K})}^{2}+m^{2}} M_{R}^{(i, j)}\left(z_{\mathscr{K}}\right), \quad f_{\mathscr{K}}^{(i, j)}= \begin{cases}1 & \text { if } v<0, \\ \prod_{l=1}^{v+1}\left(1-\frac{p_{(\mathscr{K})}^{2}+m^{2}}{p_{(\mathscr{K})}^{2}\left(z_{0}^{(l)}\right)+m^{2}}\right) & \text { if } v \geq 0\end{cases}
$$

where $\left\{z_{0}^{(l)}\right\}_{l=1}^{v+1}$ is a set of $v+1$ zeroes of the deformed amplitude $M_{n}^{(i, j)}(z)$. Although we are only including an explicit $(i, j)$ in $f_{\mathscr{K}}^{(i, j)}$, we should keep in mind that the exponent $v$ and the zeroes $z_{0}^{(l)}$ depend on the deformation as well. When $v<0$, no zeroes are needed and (2.22) is nothing but the celebrated BCFW recursion relation. Indeed BCFW need to assume that the deformed amplitude vanishes at infinity in order to derive the recursion relation. When $v \geq 0$ instead, we have a generalization of the BCFW recursion relation which can be thought of as a "weighted"11 version of BCFW (where the weights are $f_{\mathscr{K}}^{(i, j)}$ ), valid for any theory and that was first written (for massless particles) in [22]. The recursion relation may be more clear if we represent it graphically:

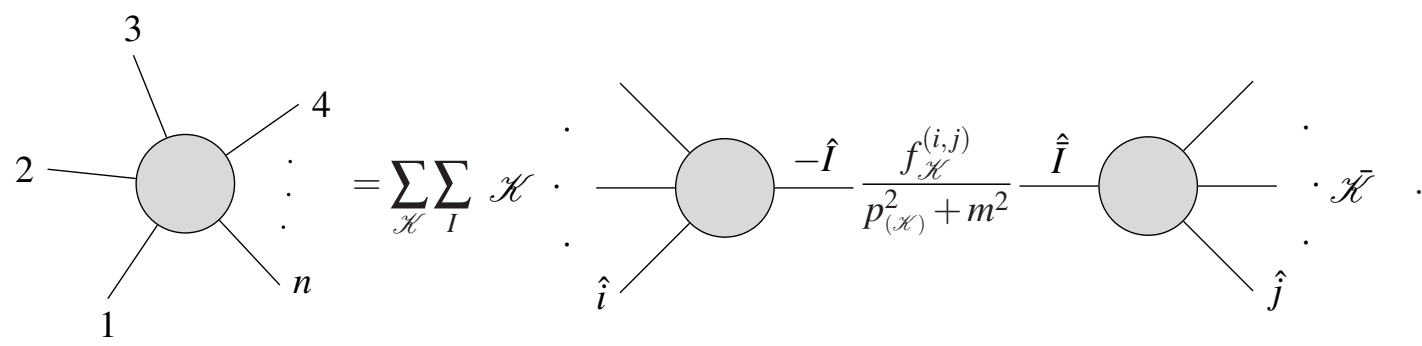

Here the hat indicates that the momenta are evaluated at $z=z_{\mathscr{K}}$. For instance, $\hat{i}$ stands for the one-particle state $\left|p_{(1)}\left(z_{\mathscr{K}}\right), a_{(1)}\right\rangle$. Recall that $\sum_{I}$ indicates a sum over all the possible internal states $-\hat{I} \equiv\left|-p_{(\mathscr{K})}\left(z_{\mathscr{K}}\right), a\right\rangle, \overline{\hat{I}} \equiv\left|p_{(\mathscr{K})}\left(z_{\mathscr{K}}\right), \bar{a}\right\rangle$ that can propagate. Some comments are in order.

Virtues of BCFW (and its generalization): The recursion relation (2.23) achieves what we were looking for, a reconstruction of the amplitude in terms of its singularities (actually just part of them). This is already an extraordinary feat, that the S-matrix program never fully realized. But one should not stop there. The BCFW recursion relation (and its generalization) provides a new representation

\footnotetext{
${ }^{11}$ Alternatively, it is appealing to think that we are deforming the propagators, an idea that people already had in mind in the 60's [1].
} 
of the scattering amplitude, alternative to the usual one via Feynman diagrams. As such, this new representation has several very (desirable) properties that the Feynman representation does not have:

- The amplitude is manifestly gauge-invariant. By this we mean that each term in the recursion relation is gauge-invariant by itself, since is made of two sub-amplitudes (which are on-shell objects, hence gauge-invariant!) and a propagator. This is to be contrasted with the Feynman representation, where each Feynman diagram is gauge-dependent, and only when we sum over all diagrams gauge invariance is recovered.

- Locality, on the other hand, is not manifest in (2.23). If we think of the Feynman representation, every Feynman diagram corresponds to a certain (off-shell) process happening in space-time. This is reflected in the singularity structure of the diagram: every singularity comes from the propagator of an off-shell particle becoming on-shell. On the contrary, the terms in the BCFW recursion relation do not correspond to real processes, as some of the momenta in the sub-amplitudes are complex! Again, in terms of singularities, each BCFW term contains "spurious" poles (i.e. poles that cannot be associated to propagating real particles) which of course cancel when summing over diagrams.

- Although we have critically used unitarity to derive (2.23), it is not manifest in the final result, in the sense that, unlike for the Feynman representation, only a subset of the factorization channels (those where particle $i$ and $j$ are in different sub-amplitudes) are explicit. The way factorization works for the missing channels is via the spurious poles we mentioned above. Incidentally, this fact is a great advantage of BCFW: while the number of possible Feynman trees grows factorially with $n$, this need not be the case for the number of BCFW terms. This makes the BCFW representation of an amplitude computationally very superior to the Feynman one. On top of that, it also explains the theoretical origin of the simplicity of certain amplitudes, like the Parke-Taylor one (see Section 2.4.2) whose BCFW representation contains one single term for any $n$.

- If the fact that we have to sum over only a subset of factorization channels were not enough, the recursive nature of (2.23) makes it even more efficient from a computational point of view. The less number of external legs, the easier the amplitude can be computed. This is also a big plus on the theoretical side: if we push the BCFW recursion relation to its smallest building block, we find that generically ${ }^{12}$ it is a three-point amplitude. No matter how many vertices we have in the Lagrangian (e.g. two for YM and infinite for GR!), the knowledge of the three-point amplitudes suffices. Furthermore, as we show in Section 2.3, these objects can be determined exactly in four dimensions, as they are fixed by the postulates of our approach. This would in principle permit to realize the S-matrix program dream of building (tree-level) amplitudes from a set of postulates. There are some obstacles to that, on which we now comment.

\footnotetext{
${ }^{12}$ Of course we can have theories where the smallest building block is a $k$-point amplitude, like the scalar theory $\lambda-\phi^{k}$. The three-point amplitude is especially appealing because it stands for a particle emitting/absorbing another one, which is the way in which we like to think that interactions occur. Indeed all known fundamental theories admit a three-point interaction.
} 
Issues and open questions: If we look back at the recursion relation we wrote in (2.22), we see that it involves three kinds of objects. One is just amplitudes (with a number of legs smaller than the total number of external particles, and with some of them carrying complex momentum), and we are familiar with them already. The other two are $v$ and $z_{0}^{(l)}$. Notice that if $v<0$, we can just take $f_{\mathscr{K}}^{(i, j)}=1$ and there is no need to take into consideration any zeroes ${ }^{13}$. Then the questions we must pose ourselves come in the following order:

- How do we determine $v$ ? This is not an easy question to analyze even from the point of view of the Feynman representation. Typically individual Feynman diagrams diverge with a power of $z$ that depends on the number of particles $n$ and has nothing to do with the power with which the total amplitude diverges. The first proofs for the vanishing at infinity of gluons [10] and graviton [23] amplitudes involved using auxiliary representations of the amplitude. A more general approach was given by Arkani-Hamed and Kaplan in [24] (later extended by Cheung [25]), who noted that the complex-UV behavior of the amplitudes, i.e. as $z \rightarrow \infty$, can be interpreted as a hard particle traveling through a soft background. If we know the Lagrangian of the theory, we can extract the coefficient $v$ (which can be made smaller than zero whenever the highest-(non-zero)spin particle of the theory is involved in the scattering).

The bad thing of all of the above is that we need a Lagrangian. From a purely S-matrix approach, there is still no completely general procedure to extract the exponent $v$ for a given theory. It has been shown in [22] that when $v>0$, it can be determined for a large class of four-dimensional theories with only massless particles (which might contain several coupling constants, but they are required to have the same dimension $d$ ):

$$
v=1-d+2 h_{(i)} \quad \text { or } \quad v=1-d-2 h_{(j)},
$$

where the $v$ we must choose depends on the factorization properties of the theory. Formula (2.24) gives the correct result for $v<0$ under certain conditions, and it has been argued in [26] why this should be the case by analyzing soft limits of three-particle amplitudes. For four-dimensional YM, $v$ has been obtained from an S-matrix approach in [27, 28].

- How do we determine the zeroes? While arguably the most interesting theories like YM or GR admit good BCFW shifts, there surely are theories like QED, scalar $\lambda-\phi^{3}$, EinsteinMaxwell, etc. where certain amplitudes do not admit them. In any case, even when a good shift is available the wrong shifts contain loop information of the theory [29], so the study of the zeroes is a relevant matter. It has been shown in [26] how to determine the zeroes of generic four-point amplitudes in four-dimensional theories with massless particles. For higher-points, they have been further studied in [30], but there is no known way to determine them generically.

\subsection{Massless kinematics in four dimensions}

So far we have been working in a generic space-time of $D+1$ dimensions, and we tried to be careful indicating when we made statements that were only valid for particular values of $D$.

\footnotetext{
${ }^{13}$ Notice though that the formula with the zeroes is still valid.
} 
Working in generic $D$ is interesting from a theoretical point of view. However, on the practical side, one is obviously interested in field theories with $D=3$. Moreover, we will further restrict to the case where the field theories only contain asymptotic massless states. This covers theories with gluons and gravitons (which are arguably the best explored theories at the theoretical level) and of course the high-energy behavior of any field theory, but leaves out other obviously relevant interactions (for instance, the ElectroWeak sector).

The main reason to focus on massless particles is simplicity, as for them we can fully exploit the so-called spinor-helicity formalism. This formalism stems from the observation that

$$
\mathrm{SO}(3,1) \cong \operatorname{SL}(2, \mathbb{C}) \text {. }
$$

More precisely it is the double cover of $\operatorname{SO}(3,1), \operatorname{Spin}(3,1)$, that is isomorphic to $\operatorname{SL}(2, \mathbb{C})$. This isomorphism can be implemented via the Pauli matrices $\tau_{a \dot{a}}^{\mu}=\left(\mathbb{1}_{a \dot{a}}, \vec{\tau}_{a \dot{a}}\right)$, to represent a null fourvector in terms of a pair of (commuting) spinors as

$$
p_{\mu} \quad \longrightarrow \quad p_{a \dot{a}}=\tau_{a \dot{a}}^{\mu} p_{\mu}=\lambda_{a} \tilde{\lambda}_{\dot{a}}
$$

The last step is possible because $\operatorname{det}\left(p_{a \dot{a}}\right)=-p^{\mu} p_{\mu}=0$. The spinors $\lambda_{a}$ and $\tilde{\lambda}_{\dot{a}}$ transform respectively in the $(\mathbf{1} / \mathbf{2}, 0)$ and $(0, \mathbf{1} / \mathbf{2})$ representation of $\operatorname{SL}(2, \mathbb{C})$. This means that under a Lorentz transformation $\Lambda$, they transform as

$$
\lambda \rightarrow e^{\frac{i}{2} \theta(\Lambda, p)} \Lambda \lambda, \quad \tilde{\lambda} \rightarrow e^{-\frac{i}{2} \theta(\Lambda, p)} \Lambda \tilde{\lambda},
$$

where $\theta(\Lambda, p)$ is the Little Group rotation angle of (1.10). They are conventionally called holomorphic and anti-holomorphic spinors respectively. In order to be more "visual", let us develop more explicitly equation (2.26):

$$
p_{\mu} \tau^{\mu}=\left(\begin{array}{cc}
p_{0}+p_{3} & p_{1}-i p_{2} \\
p_{1}+i p_{2} & p_{0}-p_{3}
\end{array}\right)=\left(\begin{array}{c}
\lambda_{1} \\
\lambda_{2}
\end{array}\right)\left(\begin{array}{ll}
\tilde{\lambda}_{i} & \tilde{\lambda}_{\dot{2}}
\end{array}\right)=\left(\begin{array}{cc}
\lambda_{1} \tilde{\lambda}_{\dot{1}} & \lambda_{1} \tilde{\lambda}_{\dot{2}} \\
\lambda_{2} \tilde{\lambda}_{\dot{1}} & \lambda_{2} \tilde{\lambda}_{\dot{2}}
\end{array}\right) .
$$

This equation has a (complex) one-parameter infinite family of solutions since the scaling

$$
\lambda \rightarrow t \lambda \quad, \quad \tilde{\lambda} \rightarrow t^{-1} \tilde{\lambda} \quad t \in \mathbb{C}
$$

preserves (2.26). That is, all the pairs of spinors in this family correspond to the same fourmomentum ${ }^{14}$. This "freedom" to choose the spinors will be very important in what is to come. A particular solution of (2.28) is

$$
\lambda_{1}=\sqrt{p_{0}+p_{3}}, \quad \lambda_{2}=\frac{p_{1}+i p_{2}}{\sqrt{p_{0}+p_{3}}} ; \quad \tilde{\lambda}=\lambda^{*} .
$$

In general we need that $\tilde{\lambda} \propto \lambda^{*}$ for $p$ to be real ${ }^{15}$. But we have seen how important it is to relax the reality condition. In the case of complex four-momenta, the isomorphism (2.25) extends to:

$$
\mathrm{SO}(3,1)_{\mathbb{C}} \cong \mathrm{SL}(2, \mathbb{C}) \times \mathrm{SL}(2, \mathbb{C}) \quad \Longrightarrow \quad \lambda \text { and } \tilde{\lambda} \text { are independent }
$$

\footnotetext{
${ }^{14}$ Because of (2.29), $\lambda$ and $\tilde{\lambda}$ are more naturally considered as elements in $\mathbb{C P}^{1}$, as in [31]. However, we will not pursue this more mathematically rigorous line here.

${ }^{15}$ This can easily be seen from the fact that the Pauli matrices are Hermitian: $\lambda_{a}^{*} \tilde{\lambda}_{\dot{a}}^{*}=p_{\mu}^{*}\left(\tau^{\mu}\right)^{*}=p_{\mu}\left(\tau^{\mu}\right)^{\top}=$ $\left(\lambda_{a} \lambda_{\dot{a}}\right)^{\top}$. Writing the components $\lambda_{a}, \tilde{\lambda}_{\dot{a}}$ in polar coordinates, one easily sees that reality requires $\tilde{\lambda} \propto \lambda^{*}$.
} 
In practice we will never use the explicit solution (2.30), which anyway is ill-defined (it is singular for momenta with components such that $p_{0}+p_{3}=0$ ); there is actually a topological obstruction to writing a continuous map from $p_{a \dot{a}}$ to $\lambda_{a}, \tilde{\lambda}_{\dot{a}}$. Instead, every operation that can be performed with the momenta has a counterpart with the spinors. The dictionary between both builds up the helicity-spinor formalism, which is just a different language than the textbook one we are typically used to (with momenta and polarization tensors). We do not need to go over all of this dictionary (the interested reader can check out e.g.: [3, 31] or the older but very complete [32]), as we are not going to compare our scattering amplitudes with the ones computed from Feynman diagrams (up to numerical factors they will be the same). This comparison is a very good exercise though! Let us present in what follows the only ingredients of the spinor-helicity formalism that will be relevant for our purposes.

It is possible to define two Lorentz-invariant inner product for spinors, one for each representation of $\operatorname{SL}(2, \mathbb{C})$ under which they can transform:

$$
\left\langle\lambda, \lambda^{\prime}\right\rangle \equiv \varepsilon^{a b} \lambda_{a} \lambda_{b}^{\prime}, \quad\left[\tilde{\lambda}, \tilde{\lambda}^{\prime}\right] \equiv \varepsilon^{\dot{a} \dot{b}} \tilde{\lambda}_{\dot{a}} \tilde{\lambda}_{\dot{b}}^{\prime}
$$

with $\varepsilon_{12}=1=\varepsilon_{12}, \varepsilon^{12}=-1=\varepsilon^{\mathrm{i} \dot{2}}$, and $\varepsilon^{a c} \varepsilon_{c b}=\delta_{b}^{a}$. These inner products are obviously antisymmetric:

$$
\left\langle\lambda, \lambda^{\prime}\right\rangle=-\left\langle\lambda^{\prime}, \lambda\right\rangle, \quad\left[\tilde{\lambda}, \tilde{\lambda}^{\prime}\right]=-\left[\tilde{\lambda}^{\prime}, \tilde{\lambda}\right]
$$

In particular, the vanishing of one of these products implies that the spinors are proportional, e.g.: $\left\langle\lambda, \lambda^{\prime}\right\rangle=0 \Longrightarrow \lambda \propto \lambda^{\prime}$. The momentum squared of a pair of light-like momenta $p_{(i)}=\lambda^{(i)} \tilde{\lambda}^{(i)}$ and $p_{(j)}=\lambda^{(j)} \tilde{\lambda}^{(j)}$ takes the form:

$$
\left(p_{i}+p_{j}\right)^{2}=2 p_{i} \cdot p_{j}=-\left\langle\lambda^{(i)}, \lambda^{(j)}\right\rangle\left[\tilde{\lambda}^{(i)}, \tilde{\lambda}^{(j)}\right] \equiv\langle i, j\rangle[j, i],
$$

where the shorthand notation in the last line is quite convenient for computational purposes. We will also use the following notation for the product of a light-like vector $p=\lambda \tilde{\lambda}$ with a generic four-momentum $P$ :

$$
2 p \cdot P=\lambda_{a} \tilde{\lambda}_{\dot{a}} P^{a \dot{a}}=\langle\lambda|P| \tilde{\lambda}] .
$$

Notice that in the particular case $P$ is light-like, (2.35) reduces to (2.34). It is quite convenient as well to know the so-called Schouten identity, which is nothing else than the fact that three complex spinors must be linearly dependent:

$$
\langle i, j\rangle \lambda^{(k)}+\langle j, k\rangle \lambda^{(i)}+\langle k, i\rangle \lambda^{(j)}=0 \quad \Leftrightarrow \quad\langle i, j\rangle\langle k, l\rangle=\langle i, l\rangle\langle k, j\rangle+\langle i, k\rangle\langle j, l\rangle .
$$

Replacing angular by square brackets, the same relation holds for the anti-holomorphic spinors. The last piece of information we will need is how to express polarization tensors in the spinorhelicity formalism. Recall that in $3+1$ dimensions, the helicity index is a semi-integer $h$, that can be plus or minus the spin of the particle. For massless particles of integer spin $s$ we have

$$
e_{a_{1} \dot{a}_{1}, \ldots, a_{s} \dot{a}_{s}}^{+}=\prod_{i=1}^{s} \varepsilon_{a_{i} \dot{a}_{i}}^{+}, \quad e_{a_{1} \dot{a}_{1}, \ldots, a_{s} \dot{a}_{s}}^{-}=\prod_{i=1}^{s} \varepsilon_{a_{i} \dot{a}_{i}}^{-},
$$

while for half-integer spin $s+1 / 2$, the polarization tensors are

$$
e_{a_{1} \dot{a}_{1}, \ldots, a_{s} \dot{a}_{s}, b}^{+}=\tilde{\lambda}_{\dot{b}} \prod_{i=1}^{s} \varepsilon_{a_{i} \dot{a}_{i}}^{+}, \quad e_{a_{1} \dot{a}_{1}, \ldots, a_{s} \dot{a}_{s}, b}^{-}=\lambda_{b} \prod_{i=1}^{s} \varepsilon_{a_{i} \dot{a}_{i}}^{-},
$$


where in the two expressions above $\varepsilon_{a \dot{a}}^{ \pm}$are polarization vectors of spin-one particles, which are given by

$$
\varepsilon_{a \dot{a}}^{+}=\frac{\mu_{a} \tilde{\lambda}_{\dot{a}}}{\sqrt{2}\langle\mu, \lambda\rangle}, \quad \varepsilon_{a \dot{a}}^{-}=\frac{\lambda_{a} \tilde{\mu}_{\dot{a}}}{\sqrt{2}[\tilde{\lambda}, \tilde{\mu}]},
$$

with $\mu_{a}$ and $\tilde{\mu}_{\dot{a}}$ arbitrary reference spinors. The presence of arbitrary reference spinors in (2.39) should not be a surprise, since as we discussed at the beginning of Section 2 polarization tensors cannot be uniquely fixed once $\{\lambda, \tilde{\lambda}, h\}$ is given. If a different reference spinor is chosen, say $\mu^{\prime}$, the for $\varepsilon_{a \dot{a}}^{+}$one can write

$$
\varepsilon_{a \dot{a}}^{+}\left(\mu^{\prime}\right)=\varepsilon_{a \dot{a}}^{+}(\mu)+\frac{\left\langle\mu^{\prime}, \mu\right\rangle}{\left\langle\mu^{\prime}, \lambda\right\rangle\langle\lambda, \mu\rangle} \lambda_{a} \tilde{\lambda}_{\dot{a}} .
$$

This is nothing but the equation (2.7) expressed in the helicity-spinor formalism.

Lorentz properties of four-dimensional massless amplitudes In the first part of the lectures we were quite careful regarding the way the Lorentz group acts on one-particle states and the Smatrix. It is worth it to particularize that knowledge to massless particles in four dimensions, using the spinor-helicity formalism.

First of all, recall that one-particle massless states are characterized by a four-momentum $p=\lambda \tilde{\lambda}$ and a helicity $h$ (and some quantum numbers $\Upsilon$ that are not important for the discussion). A massless one-particle state is then specified by the triplet $\{\lambda, \tilde{\lambda}, h\}$, and we will write the state as $|p, h\rangle=|\lambda, \tilde{\lambda}, h\rangle$. Translated to the helicity-spinor formalism, equation (1.10) becomes

$$
U(\Lambda)|\lambda, \tilde{\lambda}, h\rangle=e^{i h \theta(\Lambda, p)}\left|e^{\frac{i}{2} h \theta(\Lambda, p)} \Lambda \lambda, e^{-\frac{i}{2} h \theta(\Lambda, p)} \Lambda \tilde{\lambda}, h\right\rangle, \quad \Lambda \in \mathbb{R}^{1,3} \ltimes \operatorname{SO}(1,3) .
$$

The amplitude becomes a function of spinors and helicities:

$$
M_{n}=M_{n}\left(\left\{p_{(i)}, h_{(i)}\right\}\right)=M_{n}\left(\left\{\lambda^{(i)}, \tilde{\lambda}^{(i)}, h_{(i)}\right\}\right),
$$

and according to (1.31) it must transform in the following way:

$$
M_{n}\left(\left\{\lambda^{(i)}, \tilde{\lambda}^{(i)}, h_{(i)}\right\}\right)=e^{i\left(\sum_{i} h_{(i)}\right) \theta} M_{n}\left(\left\{e^{\frac{i}{2} \theta} \Lambda \lambda^{(i)}, e^{-\frac{i}{2} \theta} \Lambda \tilde{\lambda}^{(i)}, h_{(i)}\right\}\right) .
$$

This transformation law implies two things. First of all, in order to cancel the extra $e^{i\left(\sum_{i} h_{(i)}\right) \theta}$ phase appearing on the RHS, we can use the phases in front of the rotated Lorentz spinors of the RHS amplitude. For that we need that the amplitude scales as

$$
M_{n}\left(\left\{t \lambda^{(i)}, t^{-1} \tilde{\lambda}^{(i)}, h_{(i)}\right\}\right)=t^{-\sum_{i} 2 h_{(i)}} M_{n}\left(\left\{\lambda^{(i)}, \tilde{\lambda}^{(i)}, h_{(i)}\right\}\right) .
$$

This equation actually holds more generally, when we just scale the spinors of a single particle. Before commenting on this, notice that given the scaling (2.44), in order to satisfy the transformation rule (2.43), the amplitude can only be a function of the angular and bracket products defined in (2.32). Mixed products between holomorphic and anti-holomorphic spinors are not allowed.

Let us come back to the invariance (2.44). Consider the tensor amplitude we introduced in (2.3):

$$
M_{n}\left(\left\{\lambda^{(i)}, \tilde{\lambda}^{(i)}, h_{(i)}\right\}\right)=\prod_{i=1}^{n} e^{\mu_{1}^{(i)} \ldots \mu_{\left|h_{(i)}\right|}^{(i)}} M_{\mu_{1}^{(1)} \ldots \mu_{\left|h_{(n)}\right|}^{(n)}}\left(\left\{p_{(i)}\right\}\right) .
$$


Under the $\mathbb{C}$-transformation (2.29), the momenta are left untouched, therefore the tensor amplitude on the RHS of (2.45) does not change. This means that $M_{n}$ on the LHS must scale as the polarization tensors. It is obvious from (2.37)-(2.38) that the polarization tensor of a helicity- $h$ particle scales as $t^{-2 h}$. Therefore, taking into account that the generator of the transformation (2.29) is $\lambda_{a} \frac{\partial}{\partial \lambda_{a}}-\tilde{\lambda}_{\dot{a}} \frac{\partial}{\partial \tilde{\lambda}_{\dot{a}}}$, we arrive at the formula:

$$
\left(\lambda_{a}^{(i)} \frac{\partial}{\partial \lambda_{a}^{(i)}}-\tilde{\lambda}_{\dot{a}}^{(i)} \frac{\partial}{\partial \tilde{\lambda}_{\dot{a}}^{(i)}}\right) M_{n}\left(1^{h_{(1)}}, \ldots, n^{h_{(n)}}\right)=-2 h_{(i)} M_{n}\left(1^{h_{(1)}}, \ldots, n^{h_{(n)}}\right),
$$

where we have chosen to represent $i_{\Upsilon_{(i)}}^{h_{(i)}} \equiv\left|p_{(i)}, h_{(i)}, \Upsilon_{(i)}\right\rangle$, a notation that we will find convenient in what follows. The importance of equation (2.46) will be evidenced in the next subsection.

\subsection{Three-particle amplitudes}

As we have emphasized earlier, the recursive structure of the BCFW recursion relations is one of its main assets. The reason is that the smaller the number of external legs an amplitude has, the simpler it is to compute (this follows just from combinatorics). Thinking of the standard field-theory course, the first non-trivial amplitudes one computes are four-particle amplitudes. The reason we cannot go lower is that three-particle amplitudes are zero for massless particles with real momenta. As we have already commented in Section 1.6, this happens because it is kinematically prohibited to have a massless particle decaying into other two. However, when the momenta are complex, three-particle amplitudes are well-defined non-trivial objects! Since we have been dealing with complex momenta from a while ago, it is natural to consider these as the basic building blocks of the BCFW recursion relation ${ }^{16}$.

Furthermore, it turns out that three-point amplitudes of massless particles in four dimensions are essentially solely determined by Poincaré invariance! Their expression has been first found in [33]. We review here the derivation:

For a three-particle process, conservation of momenta implies that either the holomorphic or the anti-holomorphic spinors of the three particles are proportional.

$$
p_{(1)}+p_{(2)}+p_{(3)}=0, p_{(i)}^{2}=0 \quad \Longrightarrow \quad \begin{aligned}
& \langle 1,2\rangle[1,2]=0 \\
& \langle 3,1\rangle[3,1]=0 \\
& \langle 2,3\rangle[2,3]=0
\end{aligned} \quad \Longrightarrow \quad \begin{gathered}
\lambda_{(1)} \propto \lambda_{(2)} \propto \lambda_{(3)} \\
\text { or (/and) } \\
\tilde{\lambda}_{(1)} \propto \tilde{\lambda}_{(2)} \propto \tilde{\lambda}_{(3)}
\end{gathered}
$$

Notice that it is impossible to satisfy the conditions in the middle of (2.47) by having two proportional holomorphic spinors and two proportional anti-holomorphic ones. Then the amplitude can only be a function of angular or square brackets, so we either have

$$
M_{3}=M_{3}^{\mathrm{H}}(\langle 1,2\rangle,\langle 3,1\rangle,\langle 2,3\rangle) \quad \text { or } \quad M_{3}=M_{3}^{\mathrm{A}}([1,2],[3,1],[2,3]) .
$$

\footnotetext{
${ }^{16}$ Of course it can happen that for particular theories, like $\lambda-\phi^{4}$, the three-particle amplitude is still zero. It is conceivable that one can always treat these theories as the infinite-mass limit of another theory with non-trivial threeparticle amplitudes, in such a way that the propagation of an infinite-mass particle creates an effective higher-point vertex. This has been worked out explicitly for the aforementioned $\lambda-\phi^{4}$ in [33].
} 
Shortly we will determine which is the correct choice in terms of the helicities of the external particles. Let us suppose first that $M_{3}=M_{3}^{\mathrm{H}}$ is a function of only the holomorphic spinors. Define

$$
x_{i}=\frac{1}{2} \varepsilon_{i j k}\langle j, k\rangle
$$

Calling the helicity operator $\mathscr{H}_{i}=\lambda_{a}^{(i)} \frac{\partial}{\partial \lambda_{a}^{(i)}}-\tilde{\lambda}_{\dot{a}}^{(i)} \frac{\partial}{\partial \tilde{\lambda}_{\dot{a}}^{(i)}}$, and recalling the definition (2.32) of the inner products, one can easily check that

$$
\mathscr{H}_{i} x_{j}=\left(1-\delta_{i j}\right) x_{j} \quad \Longrightarrow \quad \mathscr{H}_{i}=\sum_{j \neq i} x_{j} \frac{\partial}{\partial x_{j}} \equiv \sum_{j \neq i} x_{j} \partial^{j} .
$$

Now we impose that the three-particle amplitude scales according to the helicity of the external particles as dictated by (2.46):

$$
\left.\begin{array}{l}
x_{2} \partial^{2} M_{3}^{\mathrm{H}}+x_{3} \partial^{3} M_{3}^{\mathrm{H}}=-2 h_{(1)} M_{3}^{\mathrm{H}} \\
x_{3} \partial^{3} M_{3}^{\mathrm{H}}+x_{1} \partial^{1} M_{3}^{\mathrm{H}}=-2 h_{(2)} M_{3}^{\mathrm{H}} \\
x_{1} \partial^{1} M_{3}^{\mathrm{H}}+x_{2} \partial^{2} M_{3}^{\mathrm{H}}=-2 h_{(3)} M_{3}^{\mathrm{H}}
\end{array}\right\} \begin{aligned}
& x_{1} \partial^{1} M_{3}^{\mathrm{H}}=\left(h_{(1)}-h_{(2)}-h_{(3)}\right) M_{3}^{\mathrm{H}} \\
& x_{2} \partial^{2} M_{3}^{\mathrm{H}}=\left(h_{(2)}-h_{(3)}-h_{(1)}\right) M_{3}^{\mathrm{H}} \\
& x_{3} \partial^{3} M_{3}^{\mathrm{H}}=\left(h_{(3)}-h_{(1)}-h_{(2)}\right) M_{3}^{\mathrm{H}}
\end{aligned}
$$

The differential equations on the right of (2.51) are homogeneous and linear, so the solution, barring delta-functions, is $M_{3}^{\mathrm{H}} \propto x_{1}^{h_{(1)}-h_{(2)}-h_{(3)}} x_{2}^{h_{(2)}-h_{(3)}-h_{(1)}} x_{3}^{h_{(3)}-h_{(1)}-h_{(2)}}$. These delta-functions are actually excluded by the cluster decomposition principle. An analogous analysis can be performed in the case $M_{3}=M_{3}^{\mathrm{A}}$. In the end we can write as the final result as

$$
\begin{aligned}
& M_{3}^{\mathrm{H}}\left(1^{h_{(1)}}, 2^{h_{(2)}}, 3^{h_{(3)}}\right)=\kappa_{\mathrm{H}}\langle 1,2\rangle^{h_{(3)}-h_{(1)}-h_{(2)}}\langle 2,3\rangle^{h_{(3)}-h_{(1)}-h_{(2)}}\langle 3,1\rangle^{h_{(2)}-h_{(3)}-h_{(1)},} \\
& M_{3}^{\mathrm{A}}\left(1^{h_{(1)}}, 2^{h_{(2)}}, 3^{h_{(3)}}\right)=\kappa_{\mathrm{A}}[1,2]^{h_{(1)}+h_{(2)}-h_{(3)}}[2,3]^{h_{(1)}+h_{(2)}-h_{(3)}}[3,1]^{h_{(3)}+h_{(1)}-h_{(2)},}
\end{aligned}
$$

where $\kappa_{\mathrm{H}}$ and $\kappa_{\mathrm{A}}$ are constants that can depend on the internal quantum numbers:

$$
\kappa_{H, A}=\kappa_{H, A}\left(\Upsilon_{(1)}, \Upsilon_{(3)}, \Upsilon_{(3)}\right)
$$

They play the role of the coupling constants of the theory. Notice that these constants must possibly change under an interchange of particles, to accommodate for the fact that the three-point amplitude flips sign if we interchange two fermions, and remains invariant in other case.

Obviously for given external helicities, the amplitude must be either holomorphic or either anti-holomorphic. In order to decide which is the correct choice, we can resort to the physical requirement that for real external momenta the three-point amplitude vanishes. In other words, the amplitude (2.52) has to go to zero as $\langle i, j\rangle$ and $[i, j]$ are both sent to zero. It is obvious that we must keep the anti-holomorphic (holomorphic) piece when $h_{(1)}+h_{(2)}+h_{(3)}>0(<0)$. The case $h_{(1)}+h_{(2)}+h_{(3)}=0$ is peculiar, as the physical requirement of the 3 -pt amplitude vanishing on the real sheet does not allow us to decide unambiguously which piece to keep. Except for the scalar case $h_{(1)}=h_{(2)}=h_{(3)}=0$, where the amplitude is just a constant (notice that such an amplitude does not vanish on the real sheet), it seems that interactions where $h_{(1)}+h_{(2)}+h_{(3)}=0$ are somewhat pathological. Indeed there are no known field theories (apart from the aforementioned scalar case) where this condition is satisfied. 
We will represent the three-particle holomorphic amplitude with a black blob, to be used when $h_{(1)}+h_{(2)}+h_{(3)}<0$, and the anti-holomorphic one with a black blob, to be used when $h_{(1)}+h_{(2)}+$ $h_{(3)}>0$. Equation (2.52) then becomes :

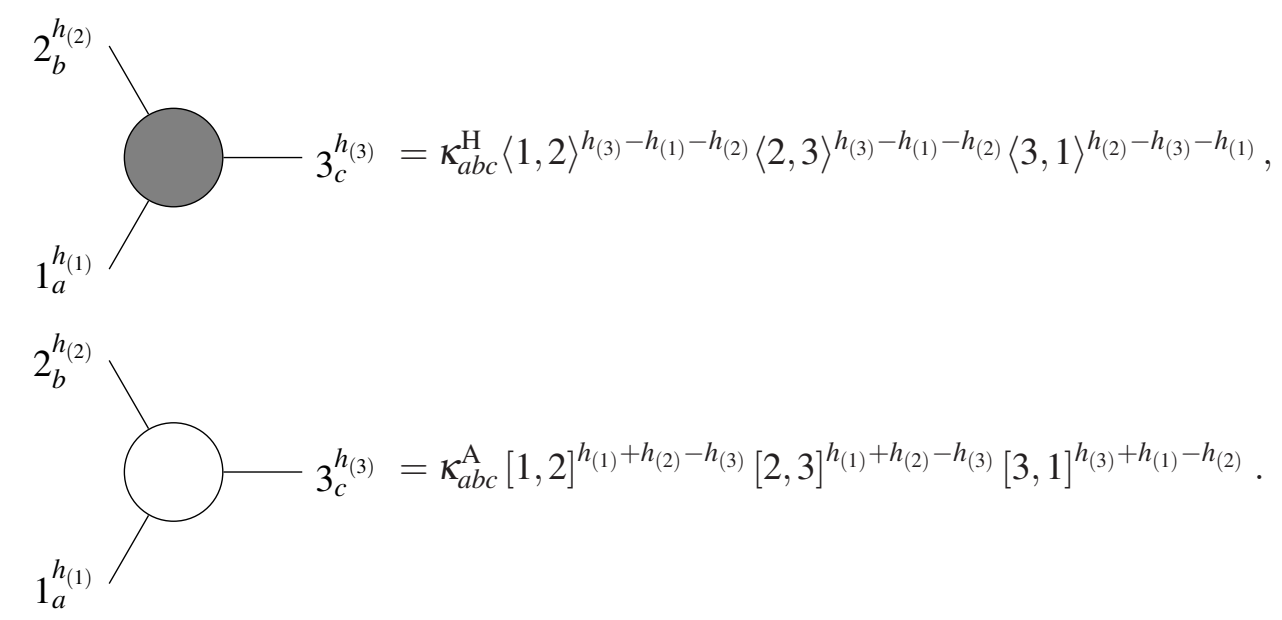

As pointed out in [33], the arguments used to obtain (2.52) are based on symmetries and make no reference to the tree level, not even to perturbation theory. So the expression for the three-particle amplitude is fully non-perturbative, and it could be used for more general purposes than the ones we have in mind here.

Before starting to use these building blocks to construct amplitudes, let us discuss some seemingly trivial, but quite important, dimensional analysis. Taking into account that the three-particle amplitude has dimensions of mass (put $D=3$ in (1.30)), it is very easy to obtain the dimension of the coupling constant:

$$
\left[\kappa_{H}\right]=\left[\kappa_{A}\right]=1-\left|h_{(1)}+h_{(2)}+h_{(3)}\right| .
$$

Imagine someone gives you the Lagrangian for the Yukawa interaction and asks you what are the helicity configurations allowed for a three-particle scattering. It is not so evident that they will be $\left(h_{(1)}, h_{(2)}, h_{(3)}\right)=\left( \pm \frac{1}{2}, \pm \frac{1}{2}, 0\right)$. However, just knowing that the coupling has to be dimensionless, it is trivial from this point of view to rule out the vertices $\left( \pm \frac{1}{2}, \mp \frac{1}{2}, 0\right)$, which would give a coupling constant with dimension of mass.

Another thing we can do with dimensional analysis is to count the number of derivatives of a vertex. Since number of derivatives is a concept attached to a Lagrangian, we have to compare the expression (2.52) with the one that would follow from a Lagrangian:

$$
M_{3} \sim \text { (coupling constant) (polarization tensors) }\left(p^{\delta}\right)
$$

where $\delta$ is the number of derivatives. Taking into account that polarization tensors are dimensionless for bosons (2.37) and have mass dimension for fermions (2.38), we can easily conclude that

$$
\delta= \begin{cases}\left|h_{1}+h_{2}+h_{3}\right| & \text { for a boson-boson-boson scattering } \\ \left|h_{1}+h_{2}+h_{3}\right|-1 & \text { for a fermion-fermion-boson scattering }\end{cases}
$$




\subsection{Three neat examples on the use of BCFW}

For the last part of the lectures, it seems a good idea to depart from the theoretical approach we have been following hitherto, and do some computations of amplitudes using BCFW so that the power of the method is more clearly exposed. We will work out three different examples illustrating different features of the method.

Before getting to it, we describe here how the BCFW deformation (2.9) looks like in the spinor-helicity formalism (recall we are working then in four dimensions and with theories with only massless particles). There are two solutions to the equations (2.11):

$$
q=\lambda^{(i)} \tilde{\lambda}^{(j)} \quad \text { or } \quad q=\lambda^{(j)} \tilde{\lambda}^{(i)} .
$$

To see that, notice that $q^{2}=0 \Longrightarrow q=\lambda \tilde{\lambda}$, and that from (2.34) it follows that $\lambda$ and $\tilde{\lambda}$ must be proportional to the spinors of $p_{(i)}$ and $p_{(j)}$ to satisfy $q \cdot p_{(i)}=0=q \cdot p_{(j)}$. The two solutions for $q$ are $q=\lambda^{(j)} \tilde{\lambda}^{(i)}$ and $q=\lambda^{(i)} \tilde{\lambda}^{(j)}$; and hence two possible BCFW shifts, that we denote as below:

$$
\begin{array}{rlrl} 
& & \\
& & & \\
& & & \\
\tilde{\lambda}^{(i)} \rightarrow \tilde{\lambda}^{(i)}(z)=\tilde{\lambda}^{(i)}-z \tilde{\lambda}^{(j)} & & \text { or } & \tilde{\lambda}^{(j)} \rightarrow \tilde{\lambda}^{(j)}(z)=\tilde{\lambda}^{(j)}-z \tilde{\lambda}^{(i)} \\
\lambda^{(j)} \rightarrow \lambda^{(j)}(z)=\lambda^{(j)}+z \lambda^{(i)} & & \lambda^{(i)} \rightarrow \lambda^{(i)}(z) & =\lambda^{(i)}+z \lambda^{(j)}
\end{array}
$$

\subsubsection{Self-interacting particle of spin $s$}

Let us consider a theory that contains only the following two three-point vertices:
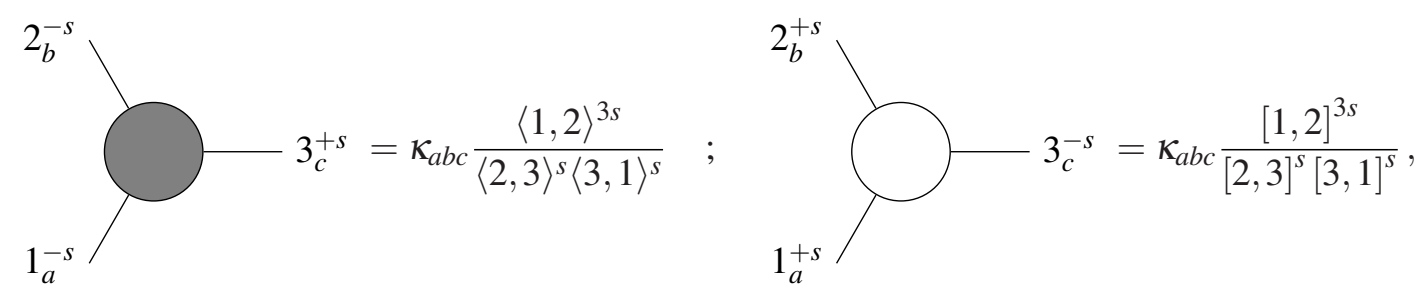

where we are allowing ${ }^{17}$ for a possible quantum number of the particle of spin $s$. The theory only contains this particle, which self-interacts as indicated in the diagrams above. It also contains only one coupling; the fact that the holomorphic and anti-holomorphic couplings are equal implies a parity-invariant interaction. Recall that the objects in (2.61) are neither Feynman diagrams nor real processes. Rather they indicate that the theory contains a three-point interaction which we will use as the building block to construct real amplitudes. The simplest non-trivial one is the four-particle amplitude, which we will compute promptly.

One should pause to remark the change in philosophy with respect to the standard QFT approach. Here the theory is defined by the particle content (the asymptotic states) and the three-point interaction. In particular, the helicity configurations allowed for this three-particle interaction determine the dimension of the coupling constant. For the particular case in $(2.61),[\kappa]=1-s$.

\footnotetext{
${ }^{17}$ Actually, for odd $s$, the quantum numbers are enforced by consistency, for the kinematic factor would flip sign under an interchange of e.g. $1 \leftrightharpoons 2$, but the amplitude must remain invariant. The only way to achieve this is by having coupling constants antisymmetric in their labels.
} 
The interaction with vertices $( \pm s, \pm s, \pm s)$ would involve a coupling constant with dimensions $[\kappa]=1-3 s$, therefore it would be a different and independent interaction. Here we are choosing the interaction $[\kappa]=1-s$ because it is what one would call the minimal-coupling interaction in a Lagrangian language. Of course we could also study a theory with the other interaction, or both.

We build the four-particle amplitude by using "inversely" the BCFW recursion relation (2.23), that is, we build the bigger amplitude "gluing" together smaller amplitudes. First of all we have to determine the possible helicity configurations of a four-particle amplitude $\left(h_{(1)}, h_{(2)}, h_{(3)}, h_{(4)}\right)$. With the three-particle amplitudes we have (2.61), and the structure of (2.23), it is easy to convince oneself that only configurations with $h_{(1)}+h_{(2)}+h_{(3)}+h_{(4)}=0$ are allowed. Without loss of generality we take then $h_{(1)}=-s=h_{(3)}, h_{(2)}=+s=h_{(4)}$. For example a helicity configuration like $(-s,+s,+s,+s)$ is not possible since it would involve gluing two anti-holomorphic amplitudes (with helicity configuration $(+s,+s,-s)$ ), but in the recursion (2.23) for four particles we have to multiply a holomorphic amplitude (where the $\tilde{\lambda}$ 's are proportional, which is possible because we deformed $\tilde{\lambda}^{(i)}$ ) times an anti-holomorphic one (where the $\lambda$ 's are proportional, which is possible because we deformed $\left.\lambda^{(j)}\right)$.

We have then agreed to study the amplitude $M_{4}\left(1_{a}^{-s}, 2_{b}^{+s}, 3_{c}^{-s}, 4_{d}^{+s}\right)$. The only thing left to discuss is which shift we choose to construct the amplitude. There is a certain freedom in this choice, to which we will come back in Section 2.4.3. Here let us choose the shift $[1,2\rangle$. It has been argued using only on-shell techniques [26] that this four-point amplitude vanishes as $z^{-s}$ under this BCFW deformation. Leaving aside for this subsection the scalar case $s=0$, we can use the standard BCFW recursion relation (2.23), where the weights are one. There are two channels to be considered:

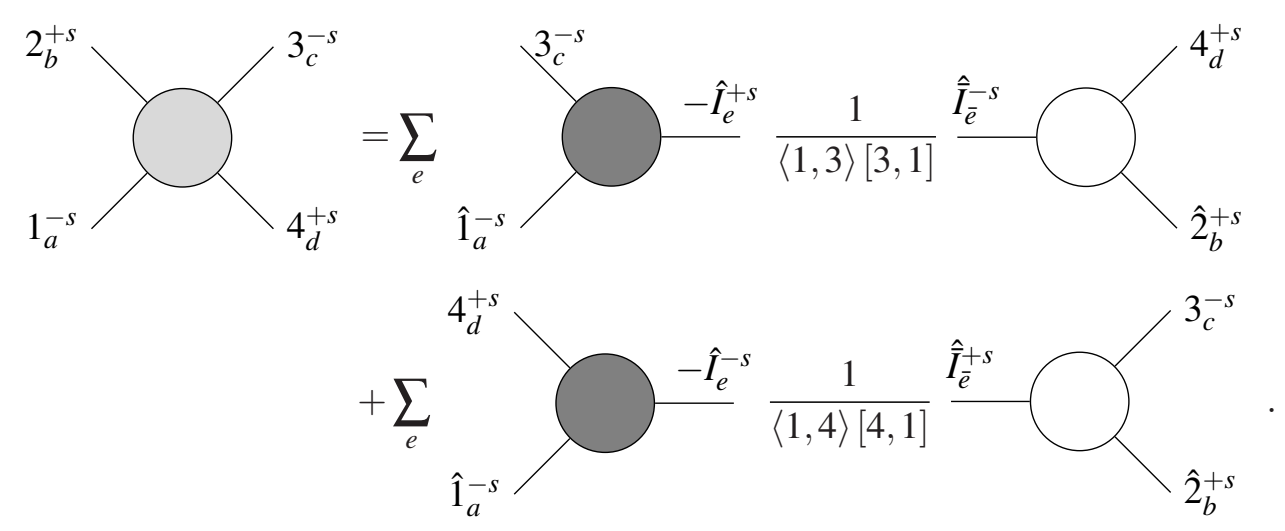

Let us work out first the u-channel ${ }^{18}$, that is, the first line in (2.62). This channel corresponds to a pole in the complex plane at a value $z=z_{(13)}$, given by

$$
0=\left(p_{(1)}\left(z_{(13)}\right)+p_{(3)}\right)^{2}=\langle 1,3\rangle\left([1,3]-z_{(13)}[2,3]\right) \quad \Longrightarrow \quad z_{(13)}=\frac{[1,3]}{[2,3]}
$$

\footnotetext{
${ }^{18}$ We define the Mandelstam variables for a four-particle process as $\mathrm{s} \equiv p_{(12)}^{2}=\langle 1,2\rangle[2,1], \mathrm{t} \equiv p_{(14)}^{2}=\langle 1,4\rangle[4,1]$ and $\mathrm{u} \equiv p_{(13)}^{2}=\langle 1,3\rangle[3,1]$.
} 
Notice that we could have said as well $0=\left(p_{(2)}\left(z_{(13)}\right)+p_{(4)}\right){ }^{2} \Longrightarrow z_{(13)}=-\frac{\langle 2,4\rangle}{\langle 1,4\rangle}$. The two expressions for $z_{(13)}$ of course coincide. This can be seen from momentum conservation:

$$
-\langle 1,4\rangle[1,3]=\left\langle 4\left|p_{(1)}\right| 3\right]=\left\langle 4\left|-p_{(2)}-p_{(4)}-p_{(4)}\right| 3\right]=-\left\langle 4\left|p_{(2)}\right| 3\right]=\langle 2,4\rangle[2,3] .
$$

Since we know the expressions of the three-particle amplitudes in terms of the spinors of the particles involved, (2.61), the only thing we have to do is to compute these spinors. For the left holomorphic blob we have:

$$
\begin{aligned}
\hat{\tilde{\lambda}}^{(1)} & =\tilde{\lambda}^{(1)}-\frac{[1,3]}{[2,3]} \tilde{\lambda}^{(2)}=\frac{[2,1]}{[2,3]} \tilde{\lambda}^{(3)}, \\
-\hat{p}_{(13)} & =\lambda^{(1)} \hat{\tilde{\lambda}}^{(1)}+\lambda^{(3)} \tilde{\lambda}^{(3)}=\left(\frac{[2,1]}{[2,3]} \lambda^{(1)}+\lambda^{(3)}\right) \tilde{\lambda}^{(3)}=\left(-\frac{\langle 3,4\rangle}{\langle 1,4\rangle} \lambda^{(1)}+\lambda^{(3)}\right) \tilde{\lambda}^{(3)} \\
& =\frac{\langle 1,3\rangle}{\langle 1,4\rangle} \lambda^{(4)} \tilde{\lambda}^{(3)} \quad \Longrightarrow \quad \hat{\lambda}^{(-I)}=\frac{\langle 1,3\rangle}{\langle 1,4\rangle} \lambda^{(4)}, \quad \hat{\tilde{\lambda}}^{(-I)}=\tilde{\lambda}^{(3)},
\end{aligned}
$$

where we have used the Schouten identity (2.36) to simplify the expressions. Recall that we have the freedom (2.29) to choose the spinors up to a complex number. This choice must not be changed along the computation in order to be self-consistent. We can then compute

$$
\bigcup_{\hat{1}_{a}^{-s}}^{3_{c}^{-s}-\hat{I}_{e}^{+s}}=\kappa_{a c e} \frac{\langle 1,3\rangle^{3 s}}{\langle 1,-\hat{I}\rangle^{s}\langle-\hat{I}, 3\rangle^{s}}=\kappa_{a c e}\left(\frac{\langle 1,3\rangle\langle 1,4\rangle}{\langle 4,3\rangle}\right)^{s}
$$

For the right anti-holomorphic blob one can follow the same steps to get:

$$
\left.\begin{array}{l}
\hat{\lambda}^{(2)}=\frac{\langle 1,2\rangle}{\langle 1,4\rangle} \lambda^{(4)} \\
\hat{\lambda}^{(\bar{I})}=\frac{\langle 1,3\rangle}{\langle 1,4\rangle} \lambda^{(4)} \\
\hat{\tilde{\lambda}}^{(\bar{I})}=\tilde{\lambda}^{(3)}
\end{array}\right\} \quad \hat{\overline{\bar{I}}}_{\overline{\bar{e}}}^{-s} \underbrace{4_{d}^{+s}}_{\hat{2}_{b}^{+s}}=\kappa_{\bar{e} d b}\left(\frac{[2,4]^{3}}{[2,3][3,4]}\right)^{s} .
$$

Multiplying (2.67) and (2.68), we have essentially computed the BCFW contribution of the uchannel to the four-particle amplitude. Let us do the same for the $t$-channel, which happens at the location $z_{(14)}=\frac{[1,4]}{[2,4]}=-\frac{\langle 2,3\rangle}{\langle 1,3\rangle}$.

$$
\begin{aligned}
& \left.\left.\begin{array}{l}
\hat{\tilde{\lambda}}^{(1)}=\frac{[2,1]}{[2,4]} \tilde{\lambda}^{(4)} \\
\hat{\lambda}^{(-I)}=\frac{\langle 1,4\rangle}{\langle 1,3\rangle} \lambda^{(3)} \\
\hat{\tilde{\lambda}}^{(-I)}=\tilde{\lambda}^{(4)}
\end{array}\right\} \quad \begin{array}{l}
4_{d}^{+s} \\
\\
\hat{1}_{a}^{-s}
\end{array}\right\}-\hat{I}_{e}^{-s}=\kappa_{\text {ade }}\left(\frac{\langle 1,4\rangle\langle 1,3\rangle}{\langle 4,3\rangle}\right)^{s} . \\
& \left.\begin{array}{l}
\hat{\lambda}^{(2)}=\frac{\langle 1,2\rangle}{\langle 1,3\rangle} \lambda^{(3)} \\
\hat{\lambda}^{(\bar{I})}=\frac{\langle 1,4\rangle}{\langle 1,3\rangle} \lambda^{(3)} \\
\hat{\tilde{\lambda}}^{(\bar{I})}=\tilde{\lambda}^{(4)}
\end{array}\right\} \quad \hat{\bar{I}}_{\bar{e}}^{+s} \underbrace{3_{c}^{-s}}_{\hat{2}_{b}^{+s}}=\kappa_{\bar{e} c b}\left(\frac{[2,4]^{3}}{[2,3][3,4]}\right)^{s} .
\end{aligned}
$$


The four-particle amplitude finally reads:

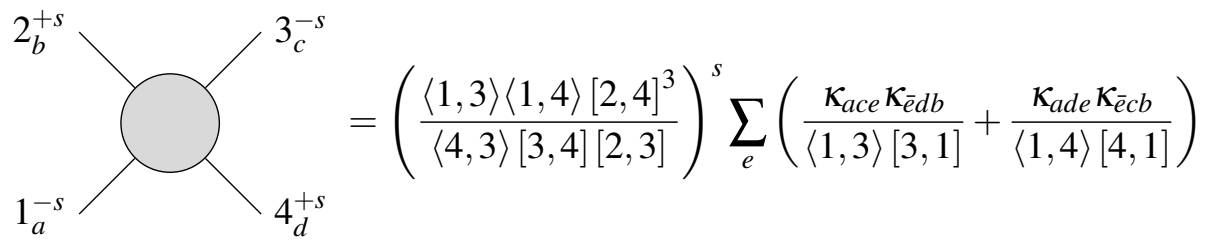

$$
\begin{aligned}
& =(-1)^{s}\left(\frac{\langle 1,3\rangle^{2}[2,4]^{2}}{\mathrm{~s}}\right)^{s} \sum_{e}\left(\frac{\kappa_{a c e} \kappa_{\bar{e} d b}}{\mathrm{u}}+\frac{\kappa_{a d e} \kappa_{\bar{e} c b}}{\mathrm{t}}\right) .
\end{aligned}
$$

\subsubsection{Parke-Taylor formula}

Arguably the Parke-Taylor formula [34] is the paramount example where the inconvenience of using Feynman diagrams for computing scattering amplitudes is exposed. Parke and Taylor computed the scattering amplitude in the YM theory for $n$ gluons in a particular helicity configuration, the so-called Maximally-Helicity-Violating (MHV) amplitude, where all but two gluons have positive helicity. They smartly re-summed a large number of Feynman diagrams, which grows exponentially with the number of external gluons $n$, to obtain a remarkably simple formula. In this subsection we revisit this computation from an on-shell perspective, obtaining very simply the Parke-Taylor result, and explaining along the way why it is so simple.

Let us focus then on YM scattering amplitudes. The external particles are spin-1 gluons. All scattering amplitudes can be constructed using the BCFW recursion relations if we one performs the shifts $[+,+\rangle,[-,-\rangle,[-,+\rangle$; in this case the amplitude vanishes at least as $z^{-1}[10,27,28]$ (for the remaining shift $[+,-\rangle$, the amplitude goes as $z^{3}$ at infinity). The three-point amplitudes are the ones in (2.61) with $s=1$ (notice that the helicity configurations in YM must be $(+,-, \pm$ ) as the coupling constant is dimensionless). In the previous section we have also computed the only non-zero four-amplitude amplitude in the theory.

A word on the MHV classification might be welcome. Given $n$ gluons to scatter, notice that it is natural, and particularly useful, to arrange the possible helicity configurations by counting the number of negative-helicity gluons we have ${ }^{19}$. It turns out that it is impossible to scatter neither $n$ positive-helicity gluons nor $n-1$ positive-helicity gluons with a negative-helicity one. A nice proof of this fact can be given using supersymmetry (at tree level, the amplitudes involving only gluons in $\mathscr{N}=4 \mathrm{SYM}$ are the same as in pure YM) [29]. Another proof, by induction, can be given immediately using the BCFW construction of YM amplitudes: we proved in the previous section that at the four-point level, only the amplitude with two pluses and two minuses (for the helicities) are allowed. To build an $n$-point amplitude with $n$ (or $n-1$ ) pluses we are forced to use smaller amplitudes with all pluses (or with all pluses but one). The result follows.

As Parke and Taylor, let us compute then the MHV amplitude, where only two gluons $i$ and $j$ have negative helicity. More concretely, Parke and Taylor computed a partial amplitude. To introduce this concept, we have to talk about:

\footnotetext{
${ }^{19}$ Of course we could count positive-helicity ones as well. By convention we call the amplitudes with only two positive-helicity gluons anti-MHV, but they are as simple as the MHV ones (one follows from the other by parity invariance actually).
} 
Color ordering. In YM it happens that the color structure of an amplitude is decoupled from its kinematical structure: $M_{n}\left(\left\{p_{(i)}, h_{(i)}, \Upsilon_{(i)}\right\}\right) \sim \sum F\left(\left\{\Upsilon_{(i)}\right\}\right) A_{n}\left(\left\{p_{(i)}, h_{(i)}\right\}\right)$. For example, for planar amplitudes (tree-level ones in particular), we can write:

$$
M_{n}\left(1_{a_{(1)}}^{h_{(1)}}, \ldots, n_{a_{(n)}}^{h_{(n)}}\right)=\sum_{\sigma} \operatorname{tr}\left(T^{a_{(\sigma(1))}} \ldots T^{a_{(\sigma(n))}}\right) A_{n}\left(\sigma(1)^{h_{(1)}}, \ldots, \sigma(n)^{h_{(n)}}\right),
$$

where we are summing over non-cyclic permutations $\sigma, T^{a}$ are the generators of the underlying $\mathfrak{s u}\left(N_{c}\right)$ Lie algebra, and $A_{n}$ is called a partial amplitude. This restricted amplitude is color-ordered, in the sense that it is computed by taking into account only color-ordered diagrams. Let us describe a simple example explaining the concept of color ordering and the origin of (2.72). All stems from the relations

$$
\left(T^{a}\right)_{j}^{i}\left(T^{a}\right)^{k}{ }_{l}=\delta_{l}^{i} \delta_{k}^{j}-\frac{1}{N_{c}} \delta_{j}^{i} \delta_{l}^{k}, \quad f^{a b c}=\operatorname{tr}\left(T^{a}\left[T^{b}, T^{c}\right]\right)
$$

From these two it follows

$$
f^{a b e} f^{e c d}=\operatorname{tr}\left(T^{a} T^{b} T^{c} T^{d}\right)-\operatorname{tr}\left(T^{b} T^{a} T^{c} T^{d}\right)-\operatorname{tr}\left(T^{a} T^{b} T^{d} T^{c}\right)+\operatorname{tr}\left(T^{b} T^{a} T^{d} T^{c}\right) .
$$

Now, imagine we are computing a four-point amplitude. Very schematically, its Feynman representation would be:

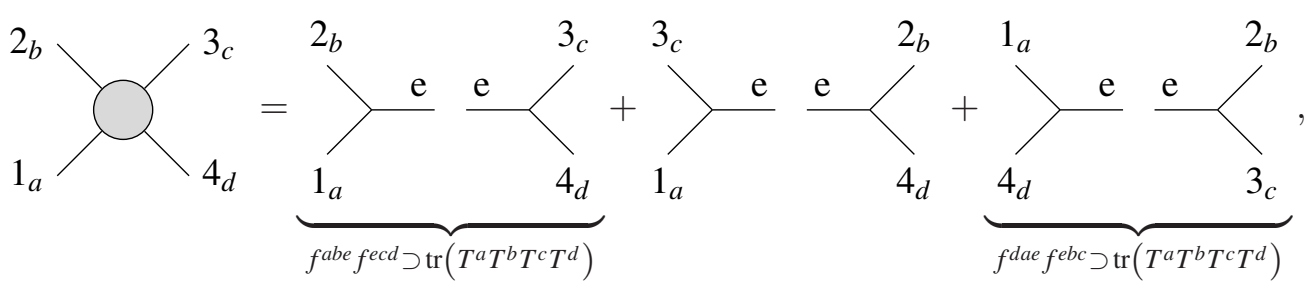

so each tree generates four traces. If we search the terms that contain the $\operatorname{trace} \operatorname{tr}\left(T^{a} T^{b} T^{c} T^{d}\right)$, we see that only the first and third tree do. This can be traced back to the fact that, reading clockwise, the labels are ordered as $(a, b, c, d)$, or any cyclic permutation, in those diagrams. The ordering is different in the second tree. Therefore, if we look for the component of the amplitude that comes with a $\operatorname{tr}\left(T^{a} T^{b} T^{c} T^{d}\right)$, we need to take into account only the diagrams where the labels are ordered as $(a, b, c, d)$ and cyclic permutations. Because each particle has a different color label, this is the same as saying that the particles in these diagrams must be ordered as $(1,2,3,4)$ and cyclic permutations.

It is easy to convince oneself that the algebra works in the same way for $n$ particles, so that if we want to extract the component of the amplitude that comes with $\operatorname{tr}\left(T^{a_{(\sigma(1))}} \cdots T^{\left.a_{(\sigma(n))}\right)}\right.$, we just need to care about the diagrams where the particles ordered as $(\sigma(1), \ldots, \sigma(n))$ and cyclic permutations. In such a case, we say the particles are color-ordered. Notice moreover that this property is combinatoric and does not depend on whether we use Feynman diagrams or other type of diagrams to represent the amplitude.

After this brief discussion of color ordering, let us get to the task of reproducing the ParkeTaylor formula:

$$
A_{n}\left(1^{+}, \ldots, i^{-}, \ldots, j^{-}, \ldots, n^{+}\right)=\frac{\langle i, j\rangle^{4}}{\langle 1,2\rangle\langle 2,3\rangle \cdots\langle n-1, n\rangle\langle n, 1\rangle}(-g)^{n+1} .
$$


Typically the Parke-Taylor formula is quoted without the $(-g)^{n+1}$ factor, as the relevant terms are the others. One should note the remarkable feature that there is only dependence on the holomorphic spinors. Let us prove the formula with BCFW via induction:

The first step of the induction is the four-point amplitude ${ }^{20} n=4$. Taking into account that we must write $\kappa_{a b c}=g f_{a b c}$ in (2.61), we can easily extract the color-ordered amplitude from (2.71). There are two partial amplitudes to consider, the one with adjacent negative-helicity particles, and the one with alternating helicities. The term with the factor $\kappa_{a d e} \kappa_{\bar{e} c b}$ in (2.71) corresponds to ordering $(a, d, c, b)$ or equivalently $(1,4,3,2)$, so we read off

$$
g^{-2} A_{4}\left(1^{-}, 4^{+}, 3^{-}, 2^{+}\right)=\frac{\langle 1,3\rangle^{2}[2,4]^{2}}{\langle 1,2\rangle[2,1]} \frac{1}{\langle 1,4\rangle[4,1]} .
$$

We exchange labels $2 \rightleftharpoons 4$ to have a standard ordering. This changes nothing in expression (2.77). We get then

$$
g^{-2} A_{4}\left(1^{-}, 2^{+}, 3^{-}, 4^{+}\right)=\frac{\langle 1,3\rangle^{3}[2,4]}{\langle 1,2\rangle\langle 3,4\rangle\langle 1,4\rangle[4,1]}=-\frac{\langle 1,3\rangle^{4}}{\langle 1,2\rangle\langle 2,3\rangle\langle 3,4\rangle\langle 4,1\rangle},
$$

which agrees with (2.76). To get the color-ordered amplitude with adjacent helicities we have to focus instead on the term with the factor $\kappa_{a c e} \kappa_{\bar{e} d b}$ in $(2.71)$. The associated ordering is $(1,3,4,2)$. Thus,

$$
g^{-2} A_{4}\left(1^{-}, 3^{-}, 4^{+}, 2^{+}\right)=\frac{\langle 1,3\rangle^{2}[2,4]^{2}}{\langle 1,2\rangle[2,1]} \frac{1}{\langle 1,3\rangle[3,1]} .
$$

Again, to recover the standard Parke-Taylor formula (2.76), we switch labels $2 \rightleftharpoons 4$, followed by $2 \rightleftharpoons 3$. This yields

$$
g^{-2} A_{4}\left(1^{-}, 2^{-}, 3^{+}, 4^{+}\right)=\frac{\langle 1,2\rangle^{2}[3,4]^{2}}{\langle 1,2\rangle[2,1]} \frac{1}{\langle 1,4\rangle[4,1]}=\frac{\langle 1,2\rangle^{2}[3,4]}{[2,3]\langle 3,4\rangle\langle 2,3\rangle}=-\frac{\langle 1,2\rangle^{3}}{\langle 2,3\rangle\langle 3,4\rangle\langle 4,1\rangle},
$$

so the first step of the induction is proved. Now let us assume that (2.76) holds up to $n-1$ particles, and prove it for $n$ external legs. Since $n \geq 5$ we can always assume (recall that cyclic permutation leaves invariant color-ordered amplitudes) that gluons 1 and $n$ have positive helicity. We then decide to use a BCFW shift $[1, n\rangle$ to compute $A_{n}$ :

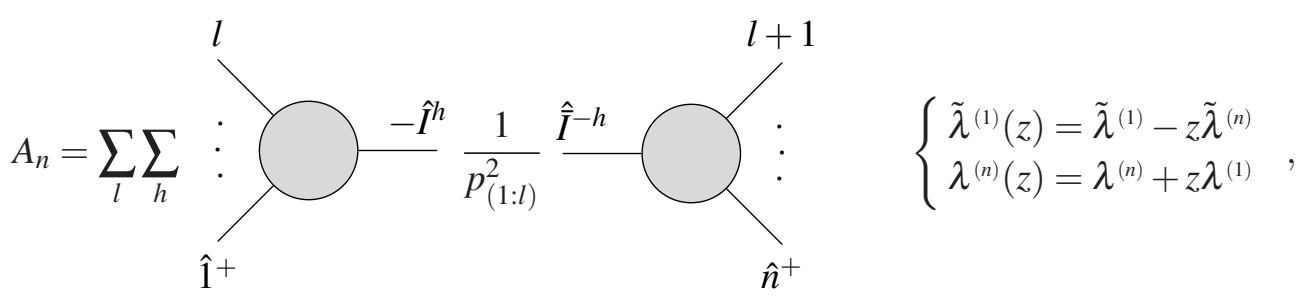

where $p_{(1: l)}=p_{(1)}+\ldots+p_{(l)}$ is the channel momentum, and $l \in\{2, \ldots, n-1\}$. For a given channel in (2.81), let us count negative-helicity gluons. We have two external ones, and one internal one. So either the left blob or the right one has only one minus. Such an amplitude is zero unless

\footnotetext{
${ }^{20}$ Actually the Parke-Taylor formula applies to $n=3$ as well: $A_{3}\left(i^{-}, j^{-}, k^{+}\right)=\frac{\langle i, j\rangle^{4}}{\langle i, j\rangle\langle j, k\rangle\langle k, i\rangle}$. We start at $n=4$ for convenience for the proof.
} 
it is a three-particle amplitude. Therefore, all but the two following channels could in principle contribute:
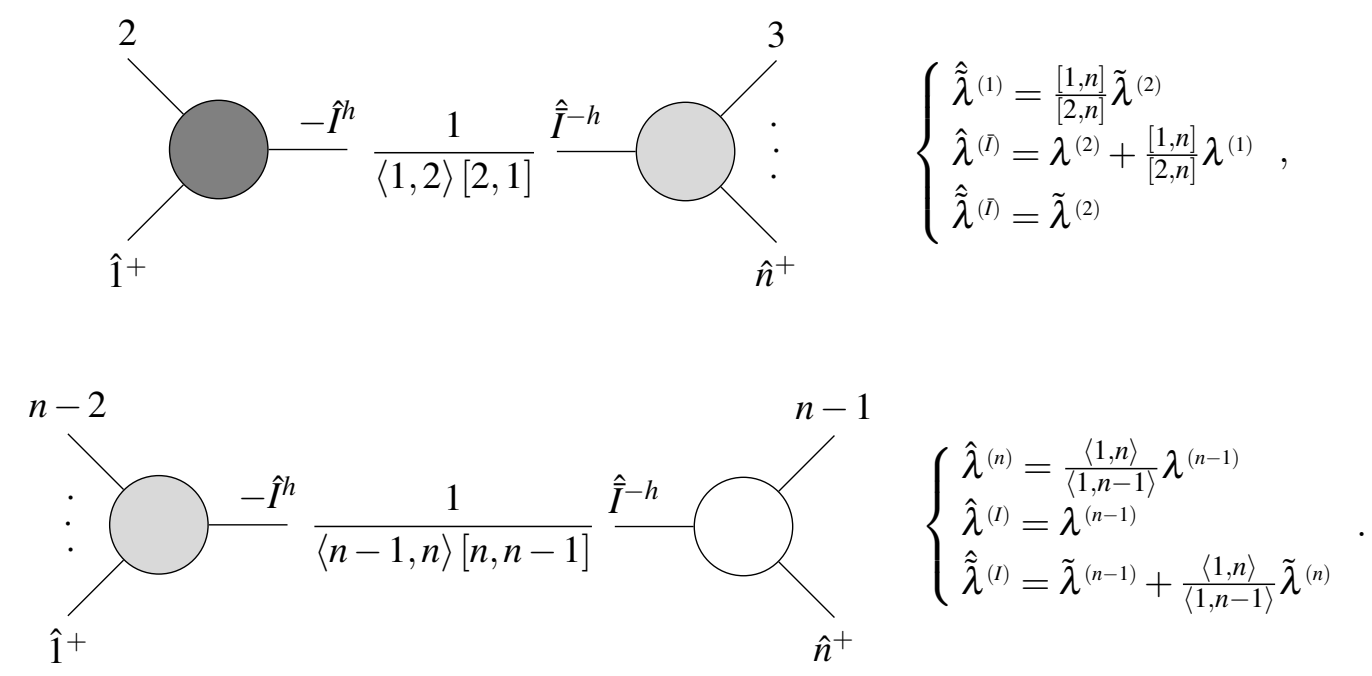

Notice that in (2.82), the left 3-pt amplitude must be holomorphic since the $\tilde{\lambda}$ 's are proportional. This implies that the two other gluons other than $\hat{1}$ must have negative helicity. Therefore, on the right sub-amplitude there is only one gluon with negative helicity. Since this amplitude contains more than three particles, it is zero. Thus, only the channel (2.83), where the right 3-pt subamplitude has to be anti-holomorphic, contributes to the total amplitude. This is the reason why the Parke-Taylor amplitude is so simple, we only have to sum over one channel! Let us do the computation.

There are two cases to be distinguished: when the gluon $n-1$ has positive helicity, and when it has negative helicity. In the first case the gluon $-I$ has to have negative helicity. Denoting by $A_{L}$ and $A_{R}$ the left and right sub-amplitudes in (2.83), we can write

$$
\begin{aligned}
A_{L} & =\frac{(-1)^{n}\langle i, j\rangle^{4}}{\langle\hat{1}, 2\rangle \cdots\langle n-2,-I\rangle\langle-I, \hat{1}\rangle}=\frac{(-g)^{n}\langle i, j\rangle^{4}}{\langle 1,2\rangle \cdots\langle n-2, n-1\rangle\langle n-1,1\rangle}, \\
A_{R} & =\frac{[n-1, \hat{n}]^{3}}{[n, \bar{I}][\bar{I}, n-1]}=g[n-1, n] \frac{\langle 1, n-1\rangle}{\langle 1, n\rangle},
\end{aligned}
$$

where we have used the fact that $A_{L}$ is an $(n-1)$-particle MHV amplitude. We immediately get

$$
A_{L} \frac{1}{\langle n-1, n\rangle[n, n-1]} A_{R}=(-g)^{n} \frac{\langle i, j\rangle^{4}}{\langle 1,2\rangle\langle 2,3\rangle \cdots\langle n-1, n\rangle\langle n, 1\rangle}(-1)^{n+1},
$$

which agrees with (2.76). In the case the gluon $n-1$ has negative helicity, we have to identify $j \equiv n-1$, and the left and right sub-amplitudes $A_{L}$ and $A_{R}$ turn out to be, after the computation, the same as in (2.84).

As we saw in this section, the Parke-Taylor formula is extremely easy to compute using the BCFW recursion relation, as opposed to its original derivation via Feynman diagrams. In hindsight, its simplicity comes from the fact that the helicity configuration is such that we can determine the amplitude with the BCFW construction by using only one of its $n$ factorization channels. 


\subsubsection{The four-particle test}

Let us do a final example of how to use BCFW to build amplitudes that beautifully exposes its power: it allows us to determine under which conditions we can define a consistent S-matrix at tree level. The idea was first introduced in [33], and refined in [26]. It follows quite naturally from the point of view we are adopting to construct amplitudes.

Let us come back to the example discussed in Section 2.4.1. There we glued three-point amplitudes to obtain four-particle ones. The gluing was done using the BCFW recursion relation. But because the BCFW method singles out two given particles, the gluing is not unique. Since different BCFW deformations will make explicit different factorization channels, it is a priori not evident that different gluings will yield the same answer. But of course, this should be the case for a consistent theory. Then, calling $M_{4}^{(i, j)}$ the object obtained when reconstructing the amplitude via an $(i, j)$-BCFW deformation, we must have

$$
M_{4}^{(i, j)}=M_{4}^{\left(i^{\prime}, j^{\prime}\right)} \quad \forall\left(i^{\prime}, j^{\prime}\right) \neq(i, j) .
$$

This condition was dubbed in [26] as the four-particle test. It turns out to impose very severe constraints on the type of three-point amplitudes we can start with to build a consistent tree level. Again, notice that the point of view we want to advocate is that of defining the theory by the asymptotic states we allow and the three-particle interactions they can have (most of the time this reduces to fixing the dimension of the coupling constant). The four-particle test highly constrains how we can mix these ingredients. Notice that this question, of writing a consistent interaction between a certain set of particles, is extremely hard to answer from the Lagrangian point of view. On the other hand, of course, having the four-particle test satisfied does not imply that we can consistently define a tree level. One could think of naturally extending this idea to an $n$-particle test for the theories that pass the four-particle one.

In the following, we will mention some impressive results that follow from the four-particle test, without going into the details of the computations (they should be straightforward at this point of the lectures). Notice that it is likely that in the computation of any of the two four-particle amplitudes in (2.86) one has to resort to the use of the zeroes in (2.22). Both the question of whether we have to use the zeroes, and its use in the case they are needed, were taken proper care of in [26] (in the original [33] it was assumed that the two amplitudes in (2.86) could be computed with the standard BCFW prescription; which turns out to be true in certain cases). Let us then comment on some applications of the four-particle test ${ }^{21}$ :

- Maybe the most straightforward application is the question of which spins are valid in the theory of the self-interacting spin-s particle that we discussed in Section 2.4.1. It turns out that we can only have $s=0, s=1$ and $s=2$. This is precisely the same conclusion as that obtained from the analysis of the soft limits of Section 1.8, that Weinberg had derived in [7]! Let us comment on each case separately:

$s=0$. The allowed theory is obviously massless $\lambda-\phi^{3}$. Notice that although this theory is illdefined because the potential is not bounded from below (at one loop, a tachyonic mass is generated), at tree level it is perfectly fine.

\footnotetext{
${ }^{21}$ See also [35] for a different, although related, on-shell way of analyzing consistency of four-particle amplitudes.
} 
$s=1$. Already at the three-point label, because of symmetry considerations we are forced to introduce structure constants. The four-particle test requires that the structure constants satisfy the Jacobi identity! Therefore, the underlying Lie algebra of YM that in the Lagrangian formalism is seen as a consequence of gauge invariance, here is just mandated by the simple consistency of four-point interactions.

$s=2$. Here we have GR. The four-particle test forbids a non-Abelian generalization of GR, a result that was known already from the standard QFT formalism [36].

- If we want to couple a particle of spin $s^{\prime}$ to gravity in such a way that the coupling has dimensions of mass ${ }^{-2}$ as the Newton constant (this is called "minimal coupling" in the Lagrangian approach), it turns out that we can only do it if $s^{\prime} \leq \frac{3}{2}$. Moreover, it also follows that the couplings have to be the same for the different spins $s^{\prime}$, which is again the quantum version of the equivalence principle derived by Weinberg with his soft-graviton theorem.

- If we look for minimal-coupling interactions of a particle of spin $s^{\prime}$ with a photon (gluon), the only allowed possibilities are QED and scalar QED (QCD and scalar QCD). The Yukawa interaction between two fermions and one scalar is also allowed.

After these examples, it should be obvious that the naive equation (2.86) is a very powerful tool. The magic here is of course performed by the BCFW recursion relations used to build the amplitudes. This representation seems to be much more insightful than the Feynman one.

\section{To loops... and beyond!}

To finish these lectures, we give a quick recap of their content and a somewhat speculative outlook of the S-matrix, or on-shell, approach to the study of QFT.

We have tried to argue how from a simple set of postulates, among which analyticity and unitarity are preeminent, one can try to recover the S-matrix of a QFT without ever displaying a Lagrangian. If we focus on the tree-level part of the S-matrix, a reasonable success can be achieved. On of the keys that has permitted this accomplishment is the BCFW recursion and related techniques, that have inspired a great deal of work along these lines. Although there are still some loose ends, it is not far-fetched to imagine that in a near future we will master the tree level from this on-shell perspective.

What about the situation at loop level? Despite many impressive works in this direction, our knowledge of the loop level from the on-shell perspective (see [37] for a reasonably recent review) is low compared to what we know about the tree level. The most successful tool to attack the computation of generic loop amplitudes is the generalized unitarity method [38], with which notable up-to six-loop computations have been performed. However, this method is not completely decoupled from the Lagrangian approach, in the sense that one needs a basis of scalar integrals to carry it out. The knowledge of such a basis, together with the regularization parameters of the integrals, come from our experience with Feynman diagrams.

Recently, a novel approach has been put forward by Arkani-Hamed, Cachazo and collaborators in a series of papers [39], that culminated in [16]. In this seminal work, the authors propose to build 
the S-matrix of planar $\mathscr{N}=4 \mathrm{SYM}^{22}$ in terms of on-shell objects called on-shell diagrams, whose combinatorics are intimately connected with the positive Grassmannian. In this new language, we have two basic building objects that we can combine in a way determined by unitarity to get any loop amplitude we want. The resulting notion of a loop is very different from the one we have in the Feynman representation. Although it is still not a very practical computational scheme, it provides a completely on-shell definition of planar $\mathscr{N}=4 \mathrm{SYM}$.

An alternative, even wilder, and connected conjectural proposal is that of the "amplituhedron" by Arkani-Hamed and Trnka [41] (which elaborates on previous observations for down-to-earth amplitudes [42]). This new formulation even departs from the vision we have advocated in these lectures, as unitarity is never demanded when building amplitudes, but it rather emerges from the geometric properties of the amplituhedron.

Since planar $\mathscr{N}=4 \mathrm{SYM}$ is very special, one can wonder if these developments can go through to more realistic theories. Certainly the understanding of UV divergencies (which are not present for $\mathscr{N}=4 \mathrm{SYM}$ ) and the renormalization group (some partial results at one loop have already been obtained [43]) from an on-shell perspective poses a magnificent challenge. This is still highly speculative arena, but one should stay tuned as new results are bound to come!

\section{Acknowledgements}

First of all, I would like to thank Pierre-Henry Lambert for the invitation and organization (without forgetting about the Dark Knights collaborating on organizational matters) of the very enjoyable Ninth Modave Summer School in Mathematical Physics. I have benefited from amiable discussions there with the participants and other lecturers of the school. Because of a bigger time overlap at the ULB department, this manuscript has particularly profited from discussions with Gustavo Lucena Gómez, Micha Moskovic and Diego Redigolo. I would like to thank as well Riccardo Argurio and Laure-Anne Douxchamps for obliging me to get my hands dirty with Lagrangians. On the non-Physics side of the school, it is a pleasure to thank again Gustavo Lucena Gómez for a fruitful partnership, Gabriele Cooontii for his ingenious retaliation skills, and in general all the crew making extra night hours at Les cent fontaines.

Finally, I would like to thank (especially) Paolo Benincasa and Freddy Cachazo for past and ongoing discussions about trying to do Physics without Lagrangians, and from whom I have learnt most of what I know about this fascinating subject.

\section{References}

[1] R.J. Eden, P.V. Landshoff, D.I. Olive, J.C. Polkinghorne, The Analytic S-matrix, Cambridge University Press 1966.

[2] S. Weinberg, The Quantum Theory of Fields. Volume I, Cambridge University Press 1995.

[3] H. Elvang and Y. -t. Huang, Scattering Amplitudes, [arXiv:1308.1697].

[4] P. Benincasa, New structures in scattering amplitudes: a review, Int. J. Mod. Phys. A 29 (2014) 5, 1430005 [arXiv:1312.5583].

\footnotetext{
${ }^{22}$ The Grasmannian construction has been extended to the ABJM theory in [40].
} 
[5] E. P. Wigner, On Unitary Representations of the Inhomogeneous Lorentz Group, Annals Math. 40 (1939) 149.

[6] P. Schuster and N. Toro, On the Theory of Continuous-Spin Particles: Wavefunctions and Soft-Factor Scattering Amplitudes, JHEP 1309 (2013) 104 [arXiv: 1302 . 1198].

[7] S. Weinberg, Photons and Gravitons in s Matrix Theory: Derivation of Charge Conservation and Equality of Gravitational and Inertial Mass, Phys. Rev. 135 (1964) B1049.

[8] S. Weinberg, Infrared photons and gravitons, Phys. Rev. 140 (1965) B516.

[9] P. Schuster and N. Toro, On the Theory of Continuous-Spin Particles: Helicity Correspondence in Radiation and Forces, JHEP 1309 (2013) 105 [arXiv: 1302.1577 ].

[10] R. Britto, F. Cachazo, B. Feng and E. Witten, Direct proof of tree-level recursion relation in Yang-Mills theory, Phys. Rev. Lett. 94 (2005) 181602 [hep-th/ 0501052$].$

[11] R. Britto, F. Cachazo and B. Feng, New recursion relations for tree amplitudes of gluons, Nucl. Phys. B715 (2005) 499 [hep-th/ 0412308 ].

[12] F. Cachazo, P. Svrcek and E. Witten, MHV vertices and tree amplitudes in gauge theory, JHEP 0409 (2004) 006 [hep-th/0403047].

[13] K. Risager, A Direct proof of the CSW rules, JHEP 0512 (2005) 003 [hep-th/ 0508206 ].

[14] T. Cohen, H. Elvang and M. Kiermaier, On-shell constructibility of tree amplitudes in general field theories, JHEP 1104 (2011) 053 [arXiv: 1010 . 0257].

[15] D. Nandan and C. Wen, Generating All Tree Amplitudes in N=4 SYM by Inverse Soft Limit, JHEP 1208 (2012) 040 [arXiv: 1204.4841$].$

[16] N. Arkani-Hamed, J. L. Bourjaily, F. Cachazo, A. B. Goncharov, A. Postnikov and J. Trnka, Scattering Amplitudes and the Positive Grassmannian, [arXiv:1212.5605].

[17] C. Boucher-Veronneau and A. J. Larkoski, Constructing Amplitudes from Their Soft Limits JHEP 1109 (2011) 130 [arXiv:1108.5385];

[18] A. Hodges, A simple formula for gravitational MHV amplitudes, [arXiv:1204.1930]; F. Cachazo and Y. Geyer, A 'Twistor String' Inspired Formula For Tree-Level Scattering Amplitudes in N=8 SUGRA, [arXiv:1206.6511]; F. Cachazo and D. Skinner, Gravity from Rational Curves in Twistor Space, Phys. Rev. Lett. 110 (2013) 16, 161301 [arXiv: 1207.0741 ]; F. Cachazo, L. Mason and D. Skinner, Gravity in Twistor Space and its Grassmannian Formulation, [arXiv:1207.4712].

[19] S. Rajabi, Higher Codimension Singularities Constructing Yang-Mills Tree Amplitudes, JHEP 1308 (2013) 037 [arXiv: 1101.5208 ]; D. C. Dunbar, J. H. Ettle and W. B. Perkins, Constructing Gravity Amplitudes from Real Soft and Collinear Factorisation, Phys. Rev. D 86 (2012) 026009 [arXiv:1203.0198].

[20] S. D. Badger, E. W. N. Glover, V. V. Khoze and P. Svrcek, Recursion relations for gauge theory amplitudes with massive particles, JHEP 0507 (2005) 025 [hep-th/ 0504159 ].

[21] K. Kampf, J. Novotny and J. Trnka, Tree-level Amplitudes in the Nonlinear Sigma Model, JHEP 1305 (2013) 032 [arXiv: 1304.3048$].$

[22] P. Benincasa and E. Conde, On the Tree-Level Structure of Scattering Amplitudes of Massless Particles, JHEP 1111 (2011) 074 [arXiv: 1106.0166 ]. 
[23] P. Benincasa, C. Boucher-Veronneau and F. Cachazo, Taming Tree Amplitudes In General Relativity, JHEP 0711 (2007) 057 [hep-th/ 0702 032].

[24] N. Arkani-Hamed and J. Kaplan, On Tree Amplitudes in Gauge Theory and Gravity, JHEP 0804 (2008) 076 [arXiv: 0801.2385$].$

[25] C. Cheung, On-Shell Recursion Relations for Generic Theories, JHEP 1003 (2010) 098 [arXiv:0808.0504].

[26] P. Benincasa and E. Conde, Exploring the S-Matrix of Massless Particles, Phys. Rev. D 86 (2012) 025007 [arXiv:1108.3078].

[27] P. Schuster and N. Toro, Constructing the Tree-Level Yang-Mills S-Matrix Using Complex Factorization, [arXiv:0811.3207].

[28] S. He and H. -b. Zhang, Consistency Conditions on S-Matrix of Spin 1 Massless Particles, JHEP 1007 (2010) 015 [arXiv:0811.3210].

[29] N. Arkani-Hamed, F. Cachazo and J. Kaplan, What is the Simplest Quantum Field Theory?, JHEP 1009 (2010) 016 [arXiv: 0808.1446 ].

[30] B. Feng, Y. Jia, H. Luo and M. Luo, Roots of Amplitudes, [arXiv: 1111.1547 ].

[31] E. Witten, Perturbative gauge theory as a string theory in twistor space, Commun. Math. Phys. 252 (2004) 189 [hep-th/0312171].

[32] L. J. Dixon, Calculating scattering amplitudes efficiently, Boulder 1995, QCD and beyond 539-582 [hep-ph/9601359].

[33] P. Benincasa and F. Cachazo, Consistency Conditions on the S-Matrix of Massless Particles, [arXiv:0705.4305].

[34] S. J. Parke and T. R. Taylor, An Amplitude for $n$ Gluon Scattering, Phys. Rev. Lett. 56 (1986) 2459

[35] D. A. McGady and L. Rodina, Higher-spin massless S-matrices in four-dimensions, [arXiv:1311.2938].

[36] N. Boulanger, T. Damour, L. Gualtieri and M. Henneaux, Inconsistency of interacting, multigraviton theories, Nucl. Phys. B597 (2001) 127 [hep-th/ 0007220$].$

[37] R. Britto, Loop Amplitudes in Gauge Theories: Modern Analytic Approaches, J. Phys. A 44 (2011) 454006 [arXiv: 1012.4493 ].

[38] Z. Bern, L. J. Dixon, D. C. Dunbar and D. A. Kosower, One loop n point gauge theory amplitudes, unitarity and collinear limits, Nucl. Phys. B425 (1994) 217 [hep-ph/ 9403226 ]; Fusing gauge theory tree amplitudes into loop amplitudes, Nucl. Phys. B435 (1995) 59 [hep-ph/ 9409265$]$;

[39] N. Arkani-Hamed, F. Cachazo, C. Cheung and J. Kaplan, The S-Matrix in Twistor Space, JHEP 1003 (2010) 110 [arXiv: 0903.2110 ]; A Duality For The S Matrix, JHEP 1003 (2010) 020 [arXiv: 0907.5418 ]; N. Arkani-Hamed, F. Cachazo and C. Cheung, The Grassmannian Origin Of Dual Superconformal Invariance, JHEP 1003 (2010) 036 [arXiv: 0909.0483 ];

N. Arkani-Hamed, J. Bourjaily, F. Cachazo and J. Trnka, Local Spacetime Physics from the Grassmannian, JHEP 1101 (2011) 108 [arXiv: 0912.3249]; Unification of Residues and Grassmannian Dualities, JHEP 1101 (2011) 049 [arXiv: 0912 . 4912]; Local Integrals for Planar Scattering Amplitudes, JHEP 1206 (2012) 125 [arXiv: 1012 . 6032]; N. Arkani-Hamed, J. L. Bourjaily, F. Cachazo, S. Caron-Huot and J. Trnka, The All-Loop Integrand For Scattering Amplitudes in Planar N=4 SYM, JHEP 1101 (2011) 041 [arXiv:1008.2958 [hep-th]]; 
[40] Y. -T. Huang and C. Wen, ABJM amplitudes and the positive orthogonal grassmannian, JHEP 1402 (2014) 104 [arXiv: 1309 .3252]; Y. -t. Huang, C. Wen and D. Xie, The Positive orthogonal Grassmannian and loop amplitudes of ABJM, [arXiv: 1402.1479].

[41] N. Arkani-Hamed and J. Trnka, The Amplituhedron, [arXiv:1312 . 2007]; Into the Amplituhedron, [arXiv:1312.7878].

[42] A. Hodges, Eliminating spurious poles from gauge-theoretic amplitudes, JHEP 1305 (2013) 135 [arXiv:0905.1473]; N. Arkani-Hamed, J. L. Bourjaily, F. Cachazo, A. Hodges and J. Trnka, $A$ Note on Polytopes for Scattering Amplitudes, JHEP 1204 (2012) 081 [arXiv: 1012 . 6030].

[43] Y. -t. Huang, D. A. McGady and C. Peng, One-loop renormalization and the S-matrix, Phys. Rev. D 87 (2013) 8, 085028 [arXiv:1205.5606 [hep-th]]. 